\title{
PD-L1 up-regulation in melanoma increases disease aggressiveness and is mediated through miR-17-5p
}

\author{
Valentina Audrito ${ }^{1,2, *}$, Sara Serra ${ }^{1,2, *}$, Aureliano Stingi ${ }^{1,2}$, Francesca Orso ${ }^{3}$, Federica \\ Gaudino $^{1,2}$, Cinzia Bologna ${ }^{1,2}$, Francesco Neri ${ }^{1}$, Giulia Garaffo ${ }^{3}$, Romina Nassini ${ }^{4}$, \\ Gianna Baroni ${ }^{5}$, Eliana Rulli ${ }^{6}$, Daniela Massi ${ }^{5}$, Salvatore Oliviero ${ }^{1,7}$, Roberto Piva $^{3}$, \\ Daniela Taverna $^{3}$, Mario Mandalà ${ }^{8, * *}$, Silvia Deaglio ${ }^{1,2, * *}$ \\ ${ }^{1}$ Human Genetics Foundation (HuGeF), Turin, Italy \\ ${ }^{2}$ Department of Medical Sciences, University of Turin, Turin, Italy \\ ${ }^{3}$ Department of Molecular Biotechnology and Health Sciences, University of Turin, Turin, Italy \\ ${ }^{4}$ Department of Health Sciences, University of Florence, Italy \\ ${ }^{5}$ Department of Surgery and Translational Medicine, University of Florence, Italy \\ ${ }^{6}$ Methodology for Clinical Research Laboratory, IRCCS - Istituto di Ricerche Farmacologiche Mario Negri, Milan, Italy \\ ${ }^{7}$ Department of Life Sciences and Systems Biology, University of Turin, Turin, Italy \\ ${ }^{8}$ Department of Oncology and Hematology, Papa Giovanni XXIII Hospital, Bergamo, Italy \\ *These authors have contributed equally to this work \\ *** These authors have shared last authorship \\ Correspondence to: Silvia Deaglio, email: silvia.deaglio@unito.it, silvia.deaglio@hugef-torino.org \\ Keywords: melanoma, targeted therapy, resistance to therapy, microRNA, regulation of gene expression \\ Received: August 11, 2016 \\ Accepted: January 06, 2017 \\ Published: February 09, 2017
}

\section{ABSTRACT}

PD-L1 is expressed by a subset of patients with metastatic melanoma (MM) with an unfavorable outcome. Its expression is increased in cells resistant to BRAF or MEK inhibitors (BRAFi or MEKi). However, the function and regulation of expression of PD$L 1$ remain incompletely understood.

After generating BRAFi- and MEKi-resistant cell lines, we observed marked upregulation of PD-L1 expression. These cells were characterized by a common gene expression profile with up-regulation of genes involved in cell movement. Consistently, in vitro they showed significantly increased invasive properties. This phenotype was controlled in part by PD-L1, as determined after silencing the molecule. Up-regulation of PD-L1 was due to post-transcriptional events controlled by miR-17-5p, which showed an inverse correlation with PD-L1 mRNA. Direct binding between miR-17-5p and the 3'-UTR of PD-L1 mRNA was demonstrated using luciferase reporter assays.

In a cohort of 80 BRAF-mutated MM patients treated with BRAFi or MEKi, constitutive expression of PD-L1 in the absence of immune infiltrate, defined the patient subset with the worst prognosis. Furthermore, PD-L1 expression increased in tissue biopsies after the metastatic lesions became resistant to BRAFi or MEKi. Lastly, plasmatic miR-17-5p levels were higher in patients with PD-L1+ than PD-L1- lesions.

In conclusion, our findings indicate that PD-L1 expression induces a more aggressive behavior in melanoma cells. We also show that PD-L1 up-regulation in BRAFi or MEKi-resistant cells is partly due to post-transcriptional mechanisms that involve miR-17-5p, suggesting that miR-17-5p may be used as a marker of PD-L1 expression by metastatic lesions and ultimately a predictor of responses to BRAFi or MEKi. 


\section{INTRODUCTION}

The therapy of metastatic melanoma (MM) was radically changed by the introduction of inhibitors of the BRAF oncogene, which is mutated in $\approx 40-50 \%$ of patients. The BRAF inhibitors (BRAFi) vemurafenib and dabrafenib were proved to be more successful than conventional chemotherapy in the treatment of these patients in terms of activity and efficacy, achieving partial and complete remissions in many instances $[1,2]$. However, resistance to BRAFi typically emerges a few months after beginning of therapy [3]. Following prolonged treatment with BRAFi, some patients also develop secondary tumors [4]. Both these phenomena are attributed to the paradoxical activation of MEK/ERK signaling consequent to the upstream block induced by BRAFi [5]. This finding was the rationale for the introduction of MEK inhibitors (MEKi) in the management of these patients [6]. The combination of BRAFi with MEKi was proposed as a strategy to delay or even prevent the onset of resistance, without increasing the risk of developing secondary cancers. Three large, prospective, randomized clinical trials indicate that combined therapy is significantly more effective than either drug used alone and that resistance occurs at a later stage, proposing this combination as the new standard treatment for this subset of MM patients [7-9].

The alternative or complementary therapeutic strategy for patients with $\mathrm{MM}$ is to restore immune functions, boosting $\mathrm{T}$ cell specific responses against the tumor [10]. Among the immune checkpoint targets of clinical importance is PD-1, which is expressed by exhausted T lymphocytes [11]. PD-1 binds to the PD-L1 ligand, which may be expressed by tumor cells, including melanoma $[12,13]$. Recent clinical trials with anti-PD-1 antibodies (nivolumab and pembrolizumab) have demonstrated higher objective response rates and increased overall survival compared to chemotherapy [14-16], albeit at the cost of significant immune-related toxicities, particularly when used in combination with anti-CTLA-4 antibodies $[17,18]$.

Regulation of PD-L1 expression by melanoma is an area of intense investigation. On the one side, PDL1 expression is induced by interferon-gamma, in turn produced by activated $\mathrm{CD} 8^{+} \mathrm{T}$ lymphocytes, highlighting immune escape mechanisms [19-22]. On the other side, PD-L1 may be induced after paradoxical activation of the MAP kinases, as recently shown [23, 24]. Furthermore, PTEN loss was found to up-regulate PD-L1, likely through the over-activation of the PI3K/Akt pathway, at least in other tumor models $[25,26]$.

In our previous work, we showed that out of a panel of 12 melanoma cell lines, A375 was the only one carrying mutations in the $B R A F$ oncogene where a distinct population of PD-L1 ${ }^{+}$cells could be defined. The sorted PD-L1 ${ }^{+}$subset of the A375 cell line was characterized by a highly invasive phenotype, with an enhanced ability to grow in xenograft models. This phenotype was attributed to the transcriptional modulation of a set of genes involved in adhesion and migration [27].

In the present work we directly link expression of PD-L1 to a more aggressive behavior of melanoma cell lines. This finding is substantiated by data obtained in patients, where intrinsic PD-L1 expression defines a subset of patients with the most unfavorable prognosis. Furthermore, we define a novel post-transcriptional circuit responsible for PD-L1 up-regulation in BRAFi-resistant melanoma cells, which is based on the direct interaction between the 3'-UTR mRNA of PD-L1 and miR-17-5p. Lastly, we show that miR-17-5p levels in patients with metastatic melanoma inversely correlate with PD-L1 expression and may predict sensitivity to BRAFi.

\section{RESULTS}

\section{Resistance to BRAFi and MEKi is accompanied by induction of PD-L1 expression in BRAF ${ }^{\mathrm{V} 600 \mathrm{E}_{-}}$ mutated melanoma cell lines}

The BRAFV600E mutated A375 (20\% of cells constitutively expressing PD-L1), SKMEL5 and M14 (both PD-L1', Figure 1A) cell lines were rendered resistant to BRAFi or MEKi by repeated exposure to increasing concentrations of each drug. Resistant cells are indicated as $\mathrm{BiR}$ and MiR, respectively. Doses were slowly escalated over a period of 12 weeks to reach a plateau of $1.6 \mu \mathrm{M}$ for both drugs. Resistance to BRAFi or MEKi was confirmed using the MTT assay (Figure 1B), as well as in xenograft models where A375/BiR, the cell line selected for in vivo experiments, failed to respond to treatment with dabrafenib, at variance with control cells (Figure 1C). No double-resistant cell line could be stabilized, at least under these experimental conditions. In these cell lines, resistance to BRAFi and MEKi was accompanied by paradoxical activation of ERK1/2 tyrosine kinase and STAT3 downstream activation (Figure 1D).

$\mathrm{MiR}$ cell lines were characterized by a robust up-modulation of CD274/PD-L1, both at the mRNA and protein levels (Figure 2A-2C). Among BiR lines, expression of PD-L1 increased in A375 (from 20\% to $100 \%$ ) and SKMEL5 (from $0 \%$ to $100 \%$ ), while M14 remained PD-L1- (Figure 2A-2C). Confocal microscopy and flow cytometry analyses confirmed that PD-L1 was intensely expressed at the cell surface (Figure 2A-2C and Figure 1A).

\section{BRAFi- and MEKi-resistant cell lines show a distinct gene profile, which partially overlaps with that of $\mathrm{A375} / \mathrm{PD}-\mathrm{L1}^{+}$cells}

We previously reported that the $\mathrm{PD}-\mathrm{L}^{+}$variant of the A375 cell line is characterized by a specific gene expression profile [27]. We now compared the genetic 
A

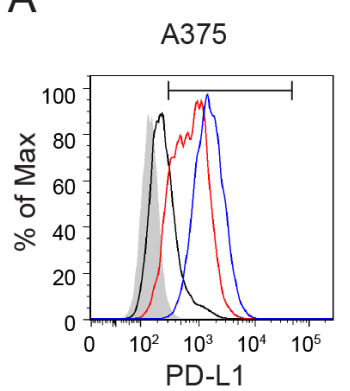

PD-L1
SKMEL5

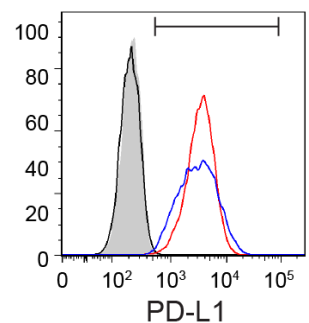

M14

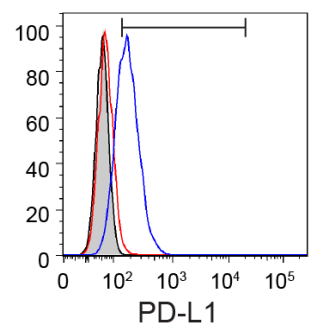

A375/PD-L1-/PD-L1+

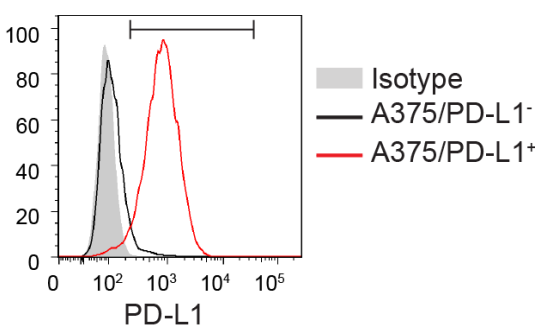

B
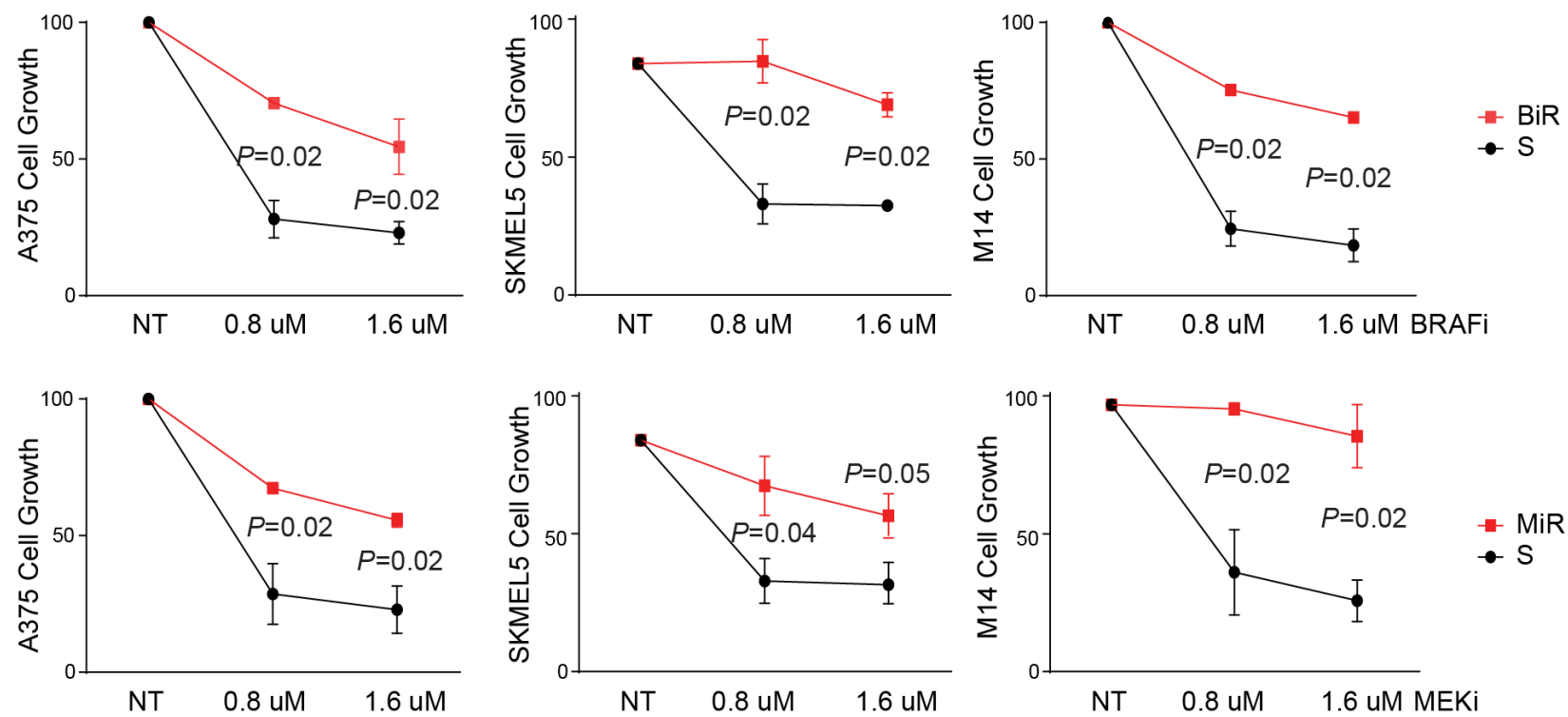

C
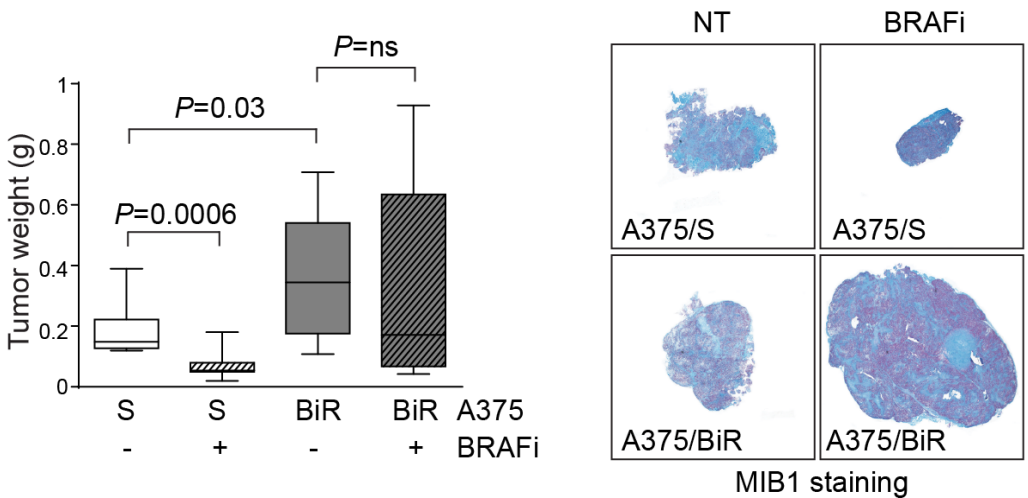

D

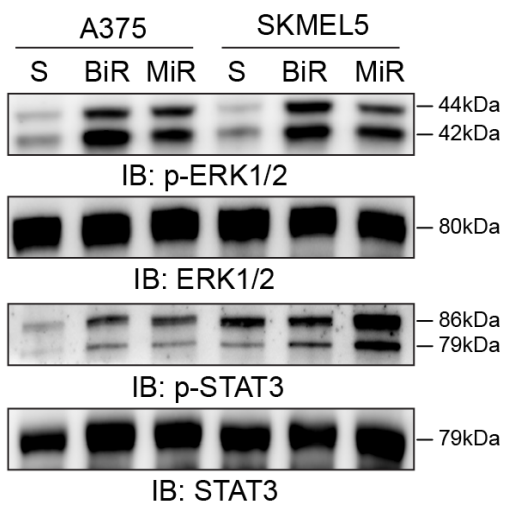

Figure 1: Establishment and characterization of BRAFV600E-mutated melanoma cell lines resistant to BRAFi and MEKi. A. Flow-cytometric histogram plots reporting surface expression of PD-L1 by A375, A375/PD-L1+, SKMEL5, M14 cell lines, before (S cells) and after the acquisition of resistance to BRAFi (BiR) and MEKi (MiR). The bars within the histogram plot define the positive cut-off. B. Cell growth of A375, SKMEL5 and M14 melanoma cell lines sensitive (black line) or resistant (red line) to BRAFi (Dabrafenib) or MEKi (Pimasertib) used at scalar doses for 72 hours as measured by MTT assay. Data are represented as \% of control (untreated cells). Data from 3 independent experiments, each performed in triplicate. C. Box plot showing tumor weight (g) of A375/S or / BiR cells after subcutaneous injection of $10^{7}$ cells in matrigel in NOD/SCID mice $(n=5)$. Cells were left to grow for 14 days before beginning treatment with BRAFi (30 mg/kg/daily gavage) for 1 week. Mice were then sacrificed and lesions stained for MIB1 to determine the proliferative fraction (representative images are shown). Original magnification x2.5 (left panels), scale bar $50 \mu \mathrm{m}$. D. Western blot analysis of p-ERK1/2 and pSTAT3, and the corresponding total protein of A375 and SKMEL5 S, BiR and MiR. S: sensitive, BiR: BRAFi resistant, MiR: MEKi resistant. 
signature of $\mathrm{A} 375 / \mathrm{BiR}$ to that of $\mathrm{A} 375 / \mathrm{PD}-\mathrm{L}^{+}$cells and found 206 commonly modulated genes (Figure $3 \mathrm{~A}$ and Supplementary Table 1). Among them, the most significantly up-modulated genes pertained to the "adhesion", "movement" and "cell growth" categories, while "antigen presentation" was the most significantly down-modulated gene category. This signature suggests that cell movement and immune escape are mechanisms shared between the A375/PD-L1 ${ }^{+}$and the A375/BiR variants (Figure 3A). We then compared the RNA sequencing profiles of $\mathrm{A} 375 / \mathrm{BiR}$ and $/ \mathrm{MiR}$ and those of SKMEL5/BiR and /MiR and identified 852 genes that were similarly modulated in the four line variants (Supplementary Table 2). Among these genes, 574 (67\%) were up-regulated and 278 (33\%) were down-regulated (Figure 3B). CD274 (PD-L1) was among the most significantly overexpressed genes in the BiR and MiR variants, confirming the validity of the approach (Figure $3 \mathrm{C})$. Gene ontology (GO) analysis confirmed that the main biological processes modulated during the acquisition of resistance concerned cell movement and immune responses (Figure 3D). Specifically, genes involved in cell adhesion, movement, signaling and immune/inflammatory responses were significantly up-regulated, while genes

A

A375

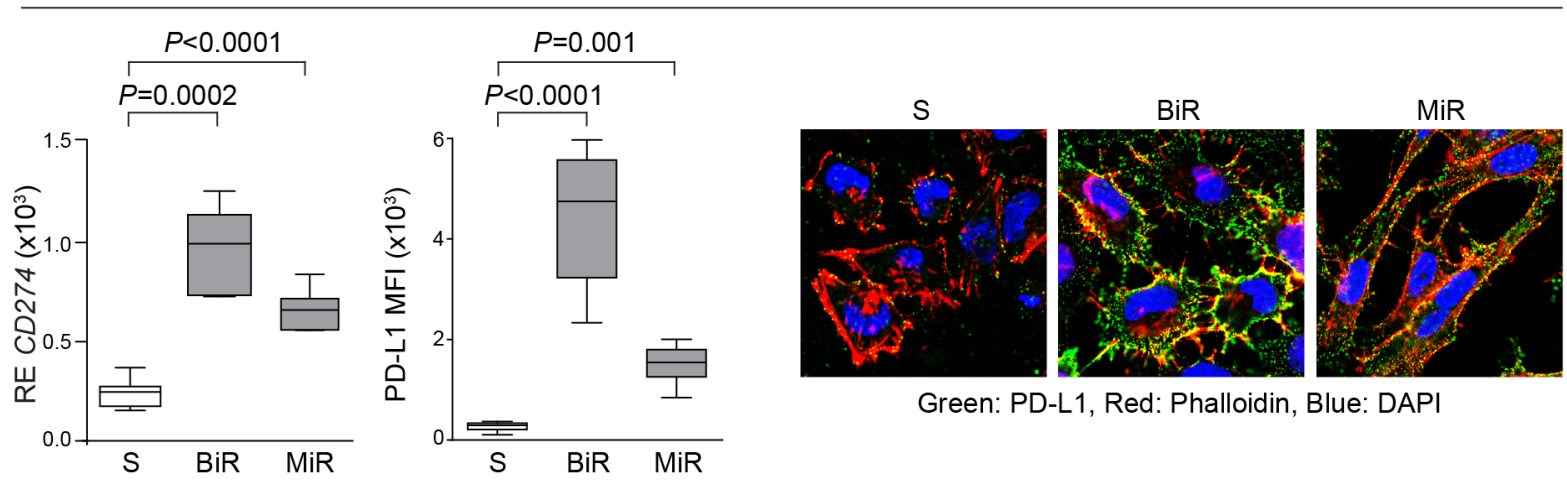

$\mathrm{B}$

SKMEL5
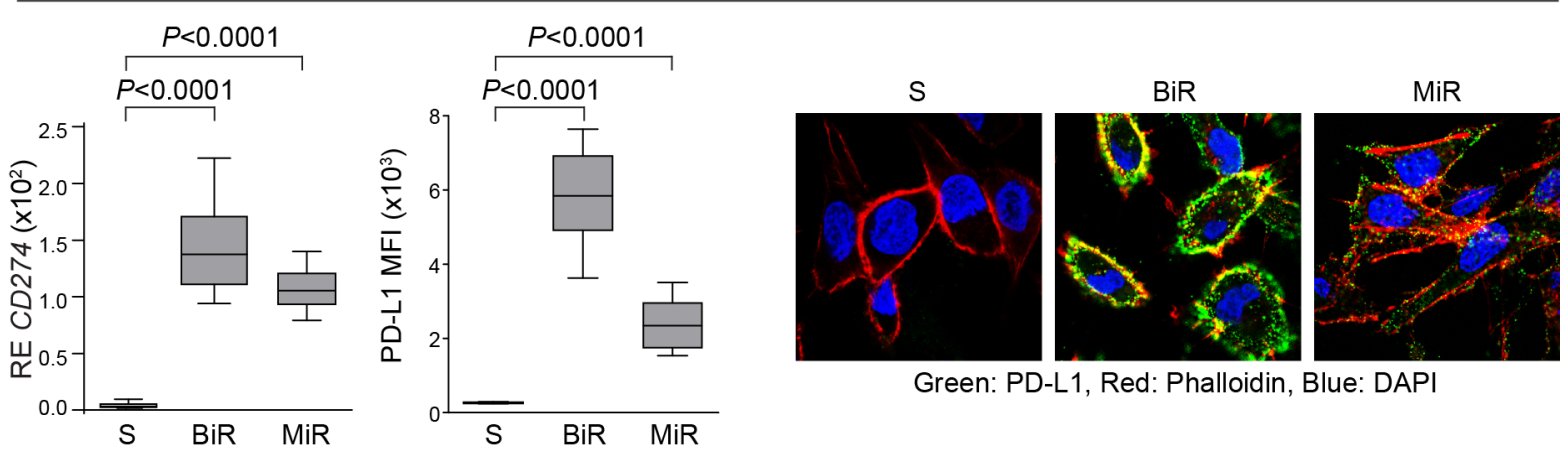

Green: PD-L1, Red: Phalloidin, Blue: DAPI

C

M14
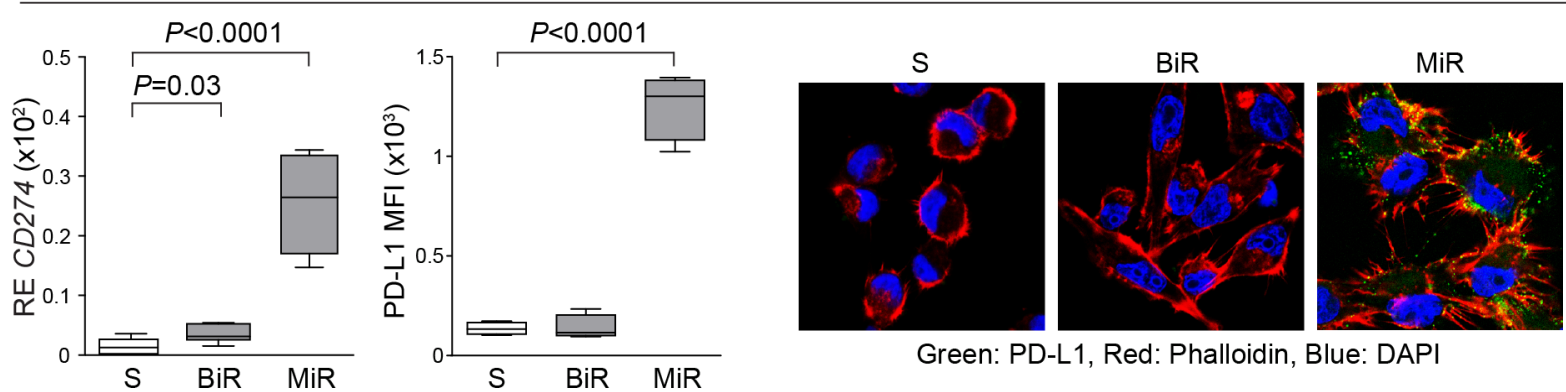

Green: PD-L1, Red: Phalloidin, Blue: DAPI

Figure 2: BRAF or MEK inhibitors resistance is accompanied by PD-L1 overexpression. A-C. Expression of $C D 274 / \mathrm{PD}-\mathrm{L} 1$ at the mRNA (left graph) and protein levels, analyzed as Mean Fluorescence Intensity (MFI, middle graph) in A375 A., SKMEL5 B. and M14 C. melanoma cell lines. Surface expression was confirmed by confocal microscopy analysis. Original magnification x63. 
A

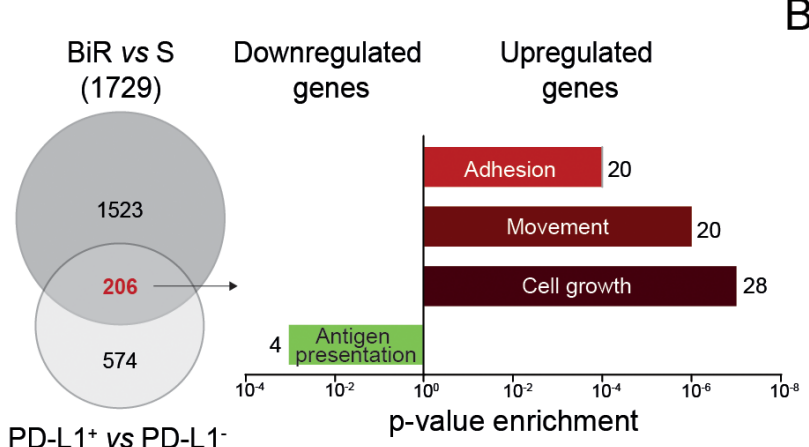

B

(780)

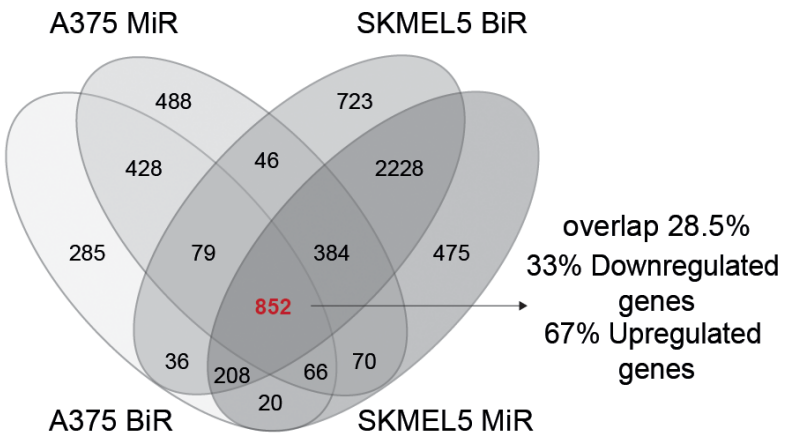

C
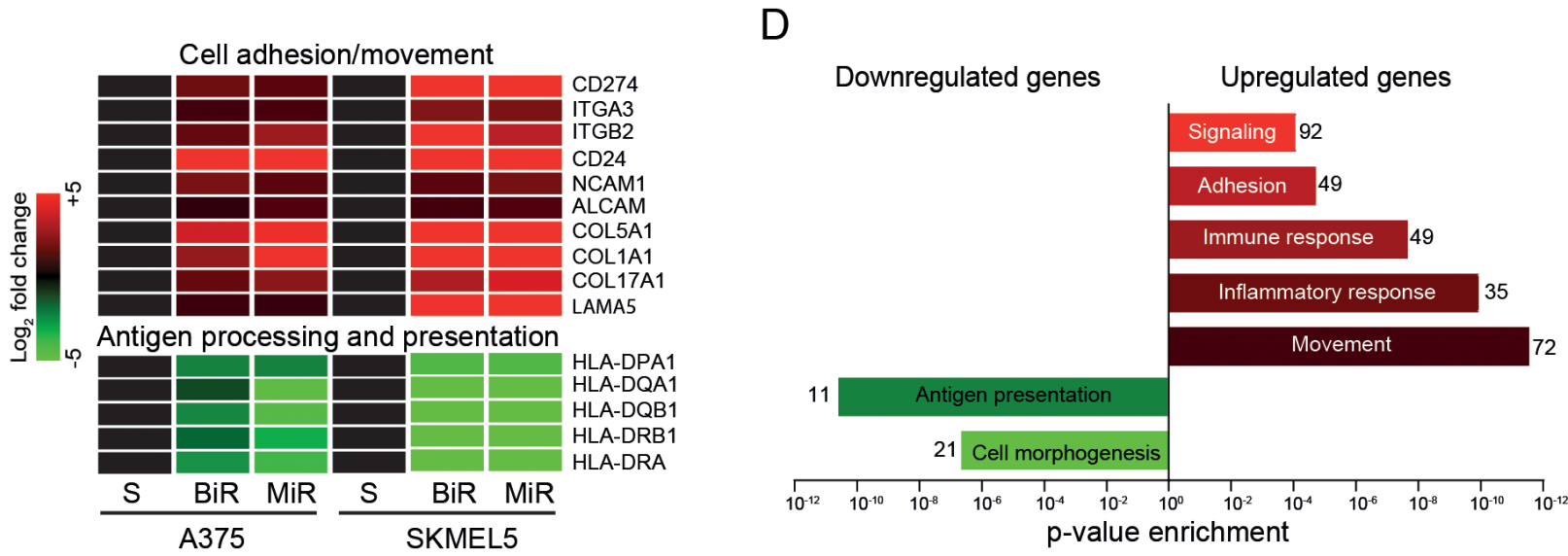

E

A375
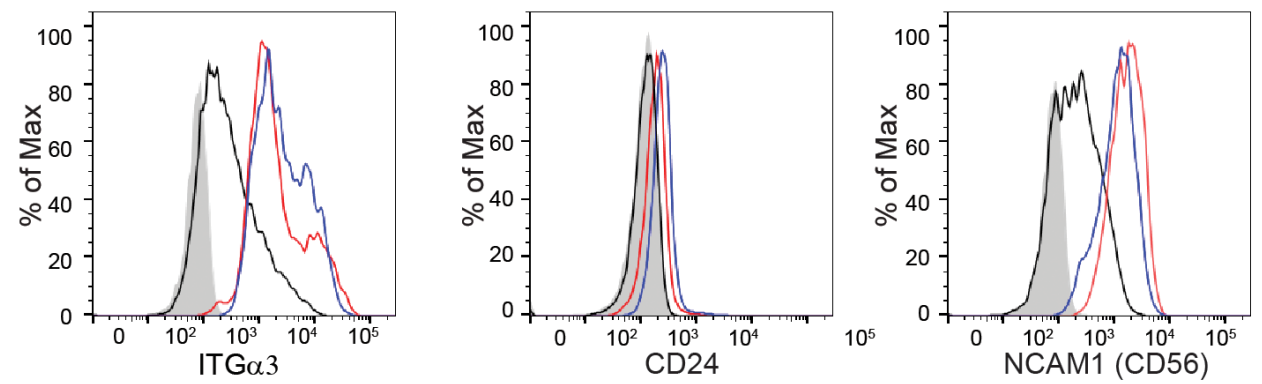

$$
\begin{aligned}
& \text { Isotype } \\
& -\mathrm{S} \\
& -\mathrm{BiR} \\
& -\mathrm{MiR}
\end{aligned}
$$
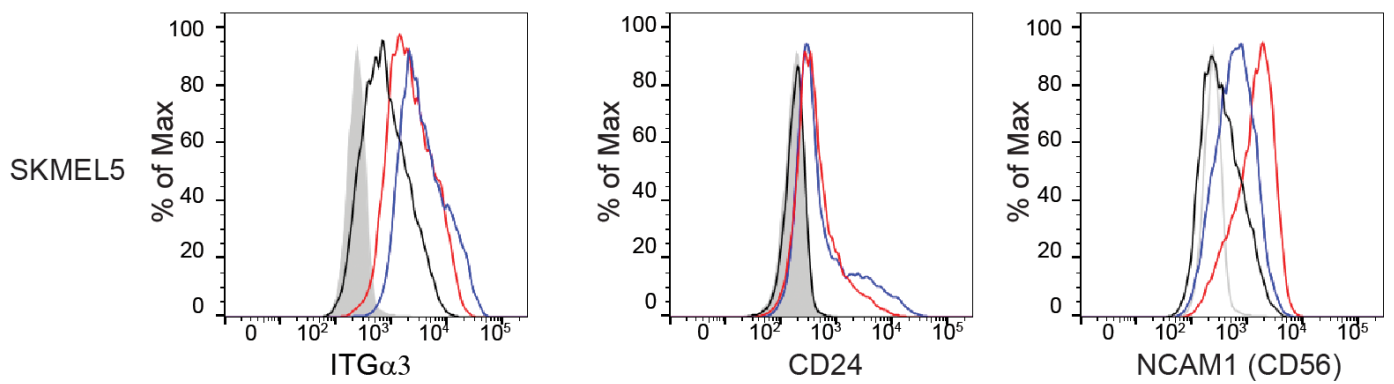

Isotype

$-S$

- BiR

$-\mathrm{MiR}$

Figure 3: PD-L1 ${ }^{+}$BRAFi or MEKi-resistant melanoma cells are characterized by a distinct genetic profile. A. Venn diagram showing genes commonly modulated when comparing A375/BiR cells to the A375/PD-L1 ${ }^{+}$clone. The histograms represent enriched gene categories obtained after analyzing the 206 common genes. B. Venn diagrams showing 852 common differentially expressed genes in BiR and MiR A375 and SKMEL5 cell lines obtained by RNAseq analysis. C. Most representative up-regulated (red) and downregulated (green) differentially expressed genes belong to the common 852 genes. D. Histograms represent the more enriched GO categories in the resistant variants. E. Differential expression of integrin- $\alpha 3$ (ITG $\alpha 3$ ), CD24 and NCAM1 (CD56) protein of sensitive and BRAFi or MEKi-resistant melanoma cells by FACS analysis. 
involved in antigen processing and presentation and cell morphogenesis were down-regulated (Figure 3C3D). Among these genes, we confirmed up-regulation of integrin $\alpha 3$ (ITG $\alpha 3$ ), CD24 and NCAM1 (CD56) at the protein level (Figure 3E), which have been previously connected to increased aggressiveness of melanoma cells. Specifically, ITG $\alpha 3$ is expressed by melanoma cells with a highly invasive potential [28], CD24 is considered a negative prognostic factor for patients with cutaneous melanoma [29], while CD56 is a neural marker, which can be expressed by melanomas with desmoplastic and spindle cell differentiation [30].

\section{Over-expression of PD-L1 in BRAFi- and MEKi- resistant cell lines contributes to increased invasiveness}

Following the gene expression profiles, we hypothesized that BiR and MiR cells would show a more aggressive behavior when compared to the drug-sensitive counterparts. Increased motility and aggressiveness of A375/PD-L1 ${ }^{+}$cells compared to A375/PD-L1- cells was previously confirmed both in vitro and in vivo [27]. We now extended these studies to A375 and SKMEL5 / $\mathrm{BiR}$ and /MiR, by analyzing their ability to repair wounds. Wound-healing assays performed at 48 hours clearly demonstrated that both /BiR and /MiR A375 and SKMEL5 cells repaired the wound in a more efficient way than the sensitive (S) counterpart (Figure 4A-4B). A375 cells showed a mean \% of repair of $21 \pm 2.5 \%$ vs $73 \pm 4 \%$ of $\mathrm{A} 375 / \mathrm{BiR}$ vs $69 \pm 3 \%$ of $\mathrm{A} 375 / \mathrm{MiR}$ (Figure $4 A-4 B)$. SKMEL5 cells showed a mean of $\%$ of repair of $15 \pm 2.5 \%$ vs $69 \pm 4 \%$ of SKMEL $5 / \mathrm{BiR}$ vs $60 \pm 9 \%$ of SKMEL5/MiR (Figure 4A-4B). Likewise BiR and MiR cells showed significantly increased chemotactic and invasive performances as compared to the $\mathrm{S}$ counterparts (Figure 4C-4D and Supplementary Figure 1). Invasion index for A375 was $3.5 \pm 5 \mathrm{SD}$ vs $193 \pm 51 \mathrm{SD}$ of A375/ $\mathrm{BiR}$ and $197 \pm 48 \mathrm{SD}$ of $\mathrm{A} 375 / \mathrm{MiR}$. Invasion index for SKMEL5 was $1.7 \pm 3$ SD vs 226 \pm 53 SD for SKMEL5/BiR and $213 \pm 50 \mathrm{SD}$ for SKMEL5/MiR (Figure 4C).

These results indicate that cells that constitutively express PD-L1 and cells that acquire PD-L1 as a consequence of the BiR state show modulation of common genes and acquire similar behavioral patterns.

\section{Silencing of PD-L1 expression influences aggressiveness}

We then asked whether PD-L1 was directly involved in determining this phenotype. To answer this question, A375/BiR or /MiR and SKMEL5/BiR or /MiR cells were infected with a lentivirus carrying a PD-L1-specific (sh) or a control shRNA (CTLR). Cells were then repeatedly selected by cloning and sorting resulting in different clones with a marked decrease in PD-L1 surface levels, as determined by flow cytometry and western blot analysis in all cell line variants (Figure 5A-5B). Silencing of PD-L1 significantly decreased the ability of A375/BiR and /MiR and of SKMEL5/BiR and /MiR cells to repair a wound (Figure 5C-5D). A375/BiR CTRL cells showed a mean $\%$ of repair of $77 \pm 9 \%$ vs $44 \pm 4 \%$ of $\mathrm{A} 375 / \mathrm{BiR}$ sh, A375/ MiR CTRL $76 \pm 6 \%$ vs $32 \pm 6 \%$ of A375/MiR sh (Figure 5C-5D). SKMEL5/BiR CTRL cells showed a mean of $\%$ of repair of $83 \pm 9 \%$ vs $62 \pm 11 \%$ of SKMEL5/BiR sh, SKMEL5/MiR CTRL $66 \pm 6 \%$ vs $37 \pm 6 \%$ of SKMEL5/MiR sh (Figure 5C-5D). Furthermore, silencing of PD-L1 in resistant cells markedly reduced the expression of CD56, and - to a lesser extent - of ITG $\alpha 3$ (Figure 5E), linking PD-L1 expression to the activation of a genetic program dictating a more aggressive phenotype, characterized by increased motility and invasion.

\section{miR-17-5p post-transcriptionally regulates $P D-$ L1 expression}

These findings underline the importance of understanding the molecular mechanisms behind PD$\mathrm{L} 1$ up-regulation in $\mathrm{BiR}$ or MiR melanoma cell lines. Previous investigators showed that PD-L1 up-regulation is dependent upon the activation of the JAK/STAT signaling pathways, in turn controlled by the MAPK pathway [23]. Consistently, BiR and MiR cell lines showed significant up-regulation of these signaling pathways (Figure 1D). However, exposure to the specific STAT3 inhibitor caused only a moderate decrease in PD-L1 expression, suggesting that other mechanisms are causing its upregulation in resistant cells [23]. For this reason, we asked whether PD-L1 up-regulation could be attributed, at least partially, to post-transcriptional mechanisms, such as those regulated by microRNAs. Based on computer predictions (TargetScan 6.0) three microRNAs, namely miR-17-5p, miR-155-5p and miR-425-5p were the only three miRs potentially targeting CD274/PD-L1 mRNA. However, only miR-17-5p showed an inverse correlation with CD274/PD-L1 mRNA levels in A375, SKMEL5 and M14 cell lines. Specifically, miR-17-5p was down-modulated in A375 and SKMEL5/BiR and /MiR variants, as well as in M14/MiR cells (Figure 6A). No significant modulation of miR-17-5p was observed in M14/BiR cells, where PD-L1 expression was unaffected (Figure 6A). Consistently, the A375/PD-L1 ${ }^{+}$variant displayed significantly lower miR17-5p levels than the PD-L1' counterpart (Figure 6A). On the contrary, miR-155-5p and miR-425-5p did not shown any apparent modulation in BiR or MiR cell lines (Supplementary Figure 2A-2B). Pearson's correlation between CD274/PD-L1 mRNA and miR-17-5p levels was $\mathrm{r}=-0.82$, with the $\mathrm{A} 375 / \mathrm{BiR}$ and $/ \mathrm{MiR}$ cells clustering together. Similar results were obtained with the SKMEL5 cells, with $\mathrm{r}=-0.62$. M14 cells behaved differently, with / BiR cells clustering with the sensitive cells, while M14/ MiR appeared to express high levels of CD274/PD-L1 
mRNA and low levels of miR-17-5p, with $r=-0.87$. This correlation was confirmed when studying miR-17-5p levels in A375/PD-L1 ${ }^{+}$and A375/PD-L1' constitutive variants, with $\mathrm{r}=-0.80$ (Figure $6 \mathrm{~B}$ ).

Transient transfection of pre-miR-17-5p in A375 and SKMEL5 /BiR and /MiR cell lines was followed by a sharp increase in intracellular miR 17-5p levels, while at the same time $C D 274 / \mathrm{PD}-\mathrm{L} 1 \mathrm{mRNA}$ levels decreased (Supplementary Figure 3A and Figure 6C). As expected, the drop in mRNA levels was accompanied by decreased in protein expression, as determined by western blot (Figure 6D) and by flow cytometry (Supplementary Figure 3B).

To determine whether miR-17-5p directly binds to the 3'-UTR region of PD-L1 we cloned part of 3'-UTR of PDL1 and expressed it in a luciferase reporter vector. We then co-transfected the reporter vector and miR-17-5p in $293 \mathrm{~T}$ cells and observed a decrease in the luciferase
A
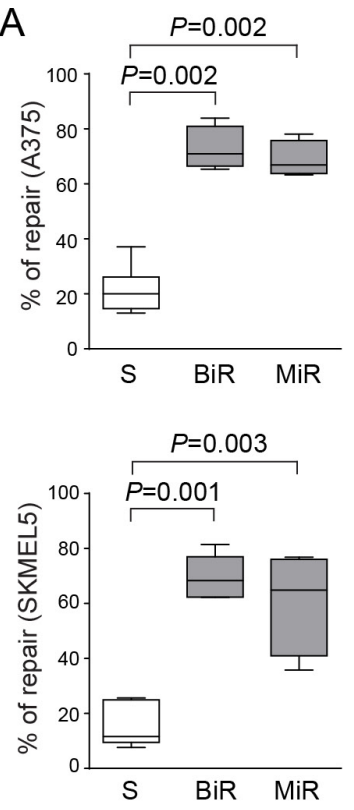

C
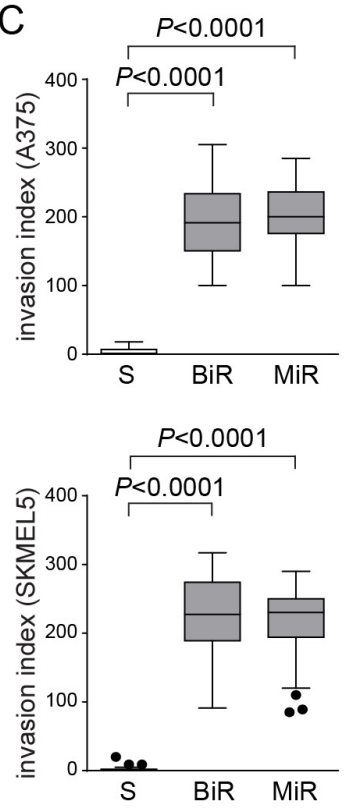

B

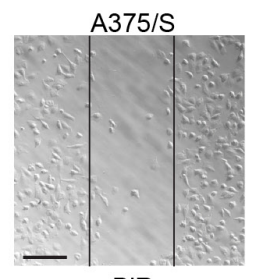

BiR
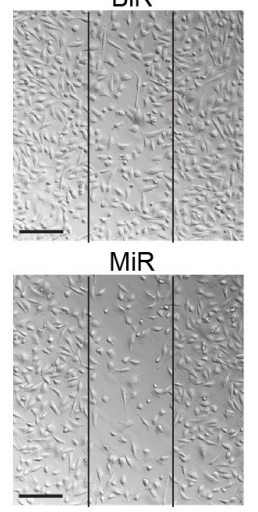

A375/S

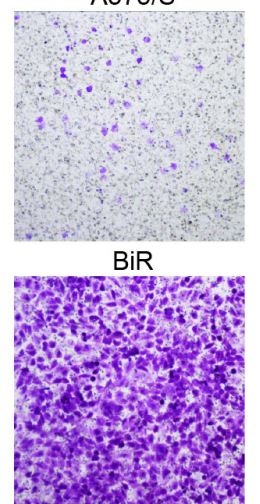

MiR

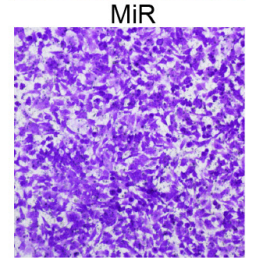

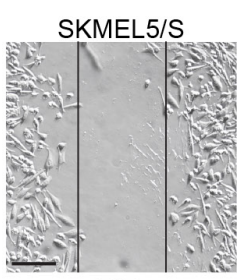

BiR

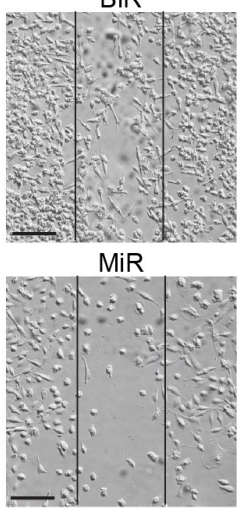

SKMEL5/S

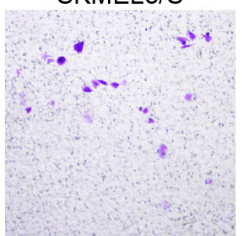

BiR

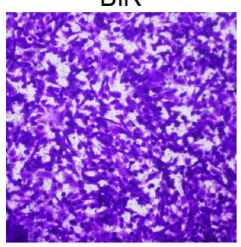

MiR

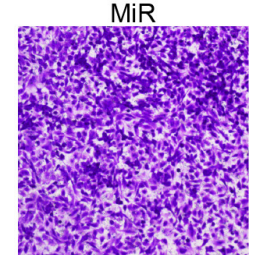

Figure 4: Over-expression of PD-L1 in BRAFi- and MEKi-resistant cell lines contributes to increased invasiveness. A. Box plots showing \% of repair in A375 and SKMEL5 variants, determined by measuring wound area ratio between two time points at 24 hours and $\mathrm{t}=0$. B. Representative pictures (x10 magnification, scale bar $200 \mu \mathrm{m}$ ) of wound-healing assay comparing S, BiR and MiR variants. C. Box plots representing cumulative data of invasion assays performed using A375 and SKMEL5 cell variants. D. Representative images (x10 magnification) of A375 (left panels) and SKMEL5 (right panels) cell lines comparing S, BiR and MiR variants. 
A

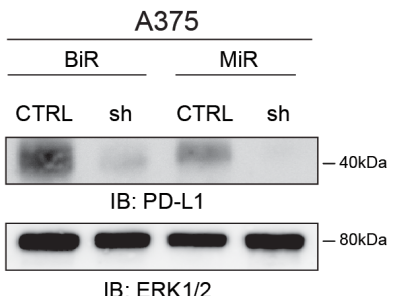

B

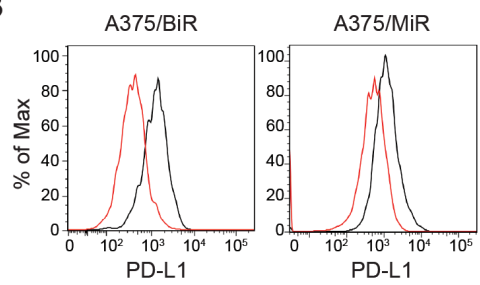

— CTRL - sh

C
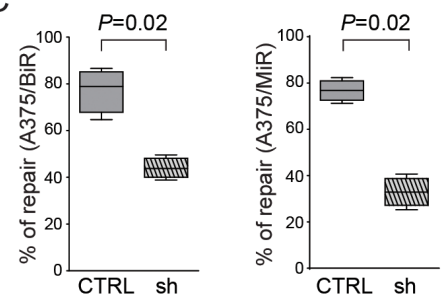

D
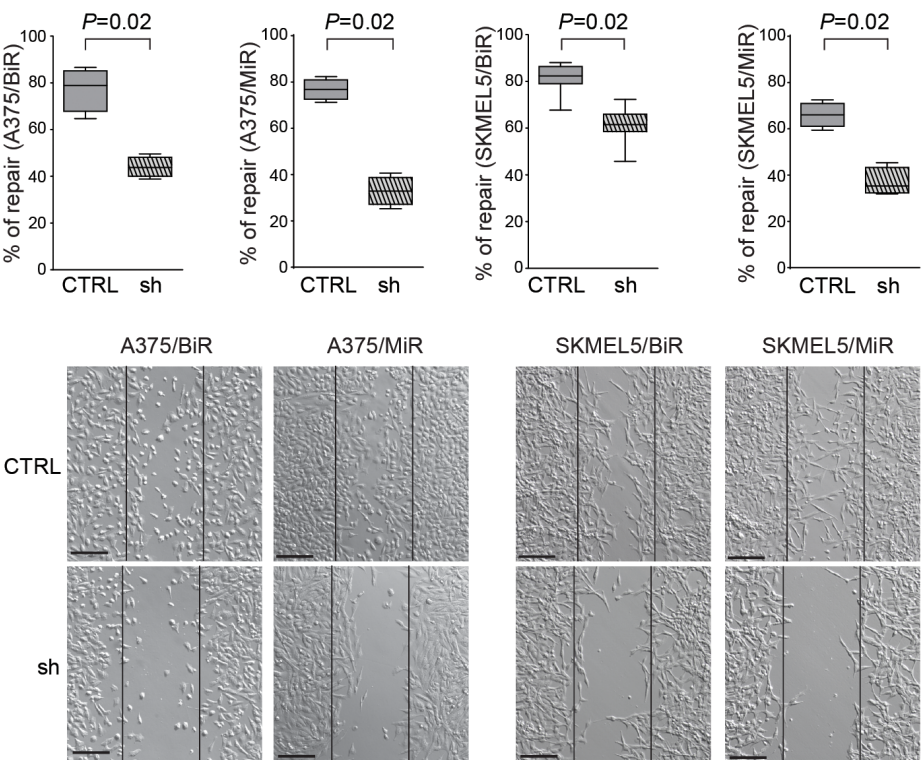

E

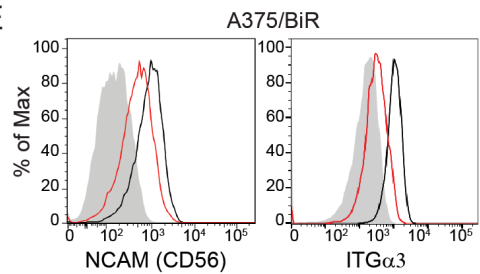

SKMEL5/BiR

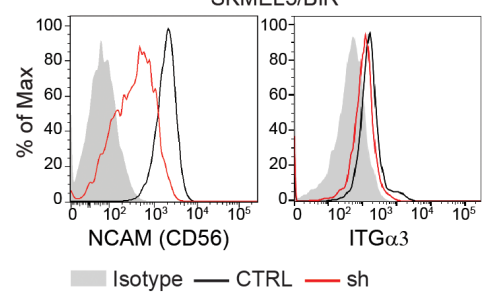

SKMEL5

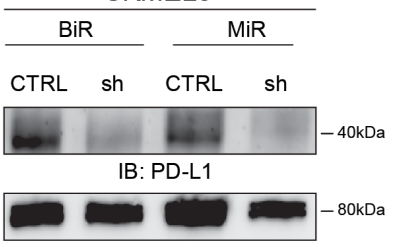

IB: ERK1/2
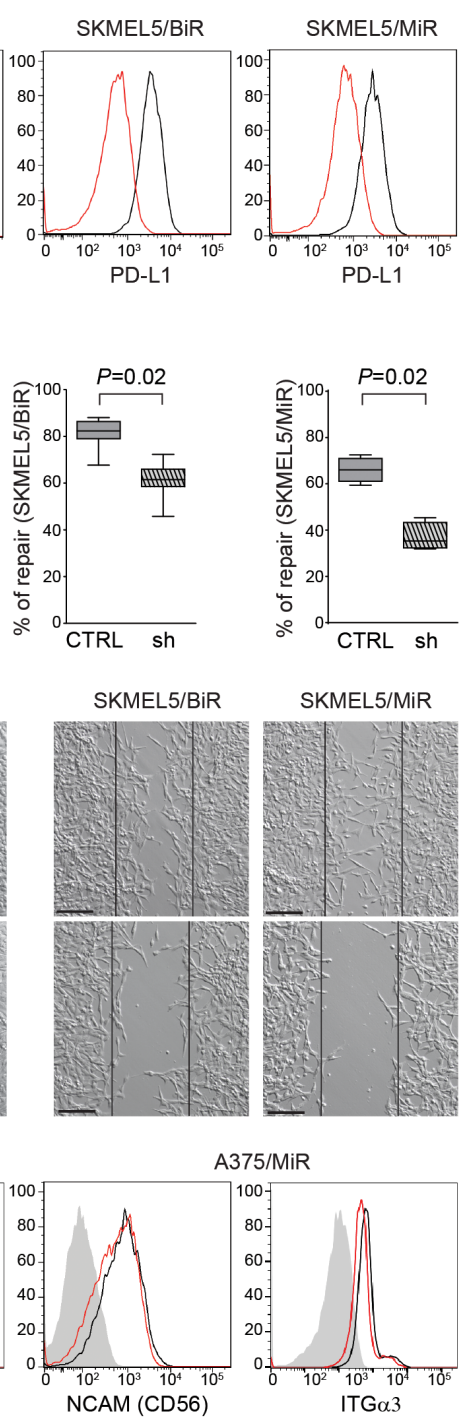

SKMEL5/MiR

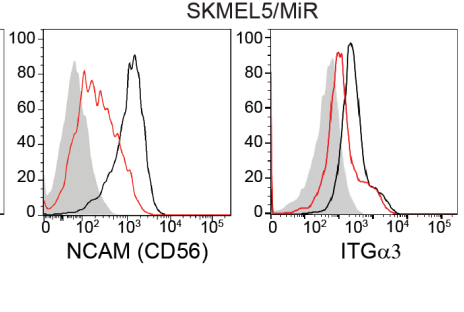

Figure 5: PD-L1 silencing influences aggressiveness. A. PD-L1 protein level of silenced infected cells (sh) in comparison to the infected control (CTRL) in A375/BiR, A375/MiR, SKMEL5/BiR and SKMEL5/MiR cells. B. Flow-cytometric histogram plots of PD-L1silenced A375 and SKMEL5 resistant cell lines. C. Box plot of wound healing repair of A375/BiR, A375/MiR, SKMEL5/BiR, SKMEL5/ MiR CTRL $v s$ sh. D. Representative pictures (x10 magnification, scale bar $200 \mu \mathrm{m}$ ) of wound-healing assay comparing the infected control (CTRL) versus the infected (sh) in A375/BiR, A375/MiR and SKMEL5/BiR and SKMEL5/MiR cells. E. Histogram representing CD56 and ITGa3 surface protein expression in CTRL and sh A375/BiR and SKMEL5/BiR cells. 

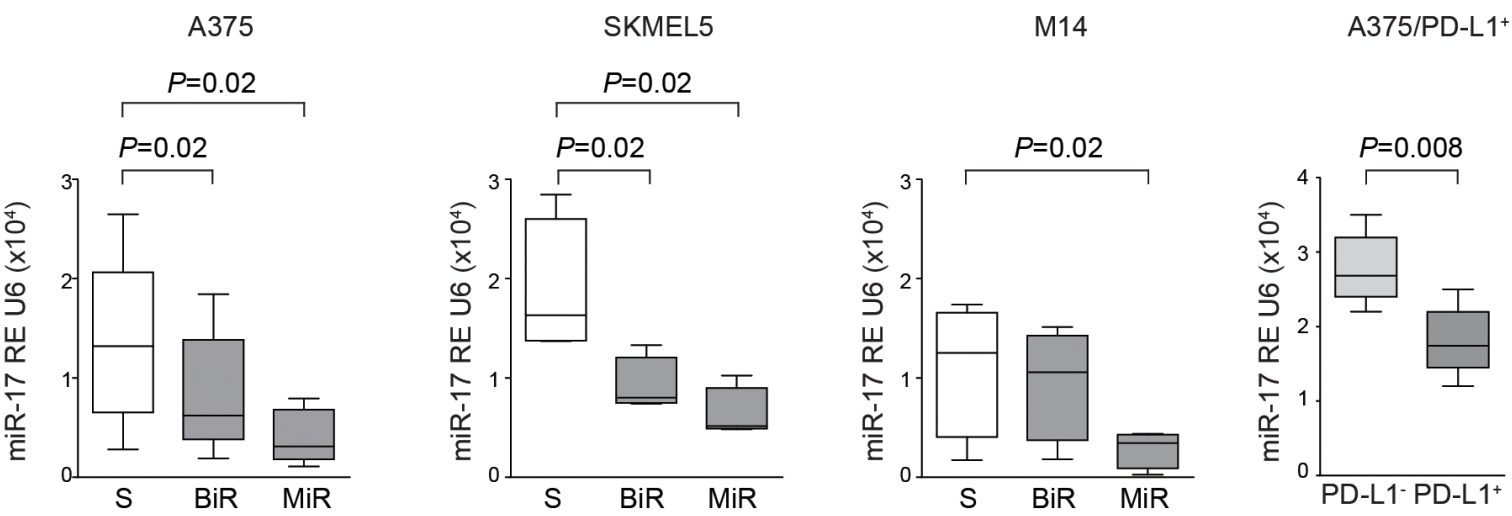

B
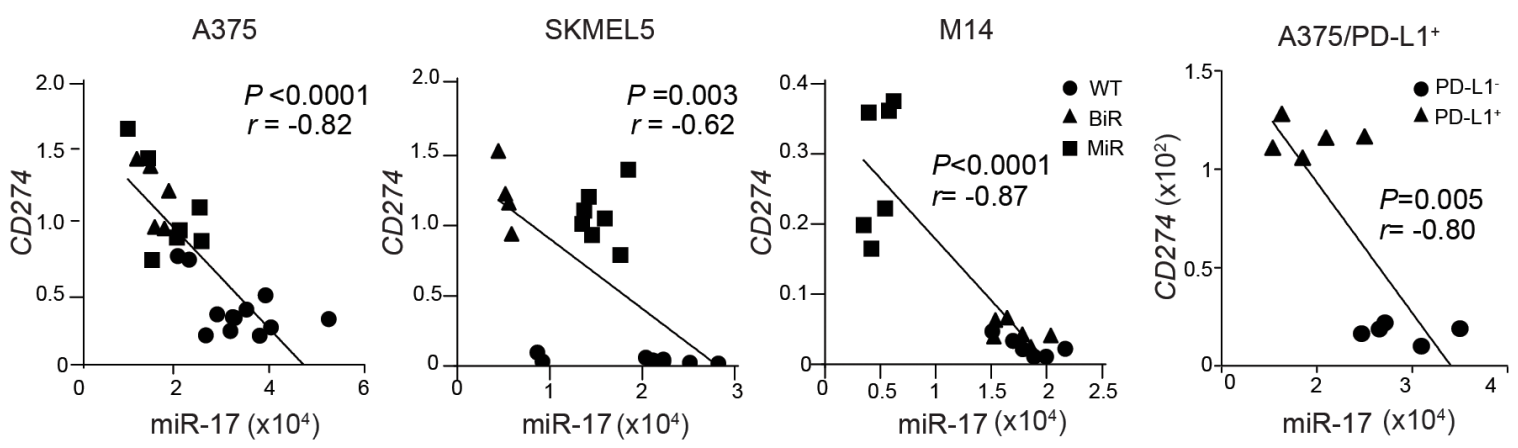

C
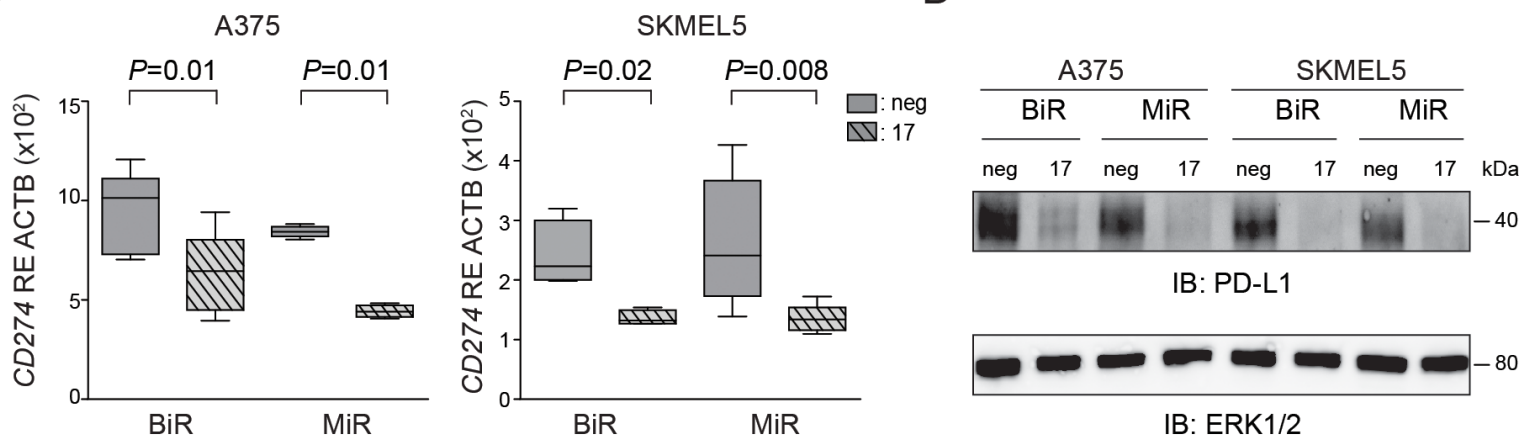

IB: PD-L1

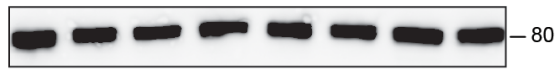

$E$

F
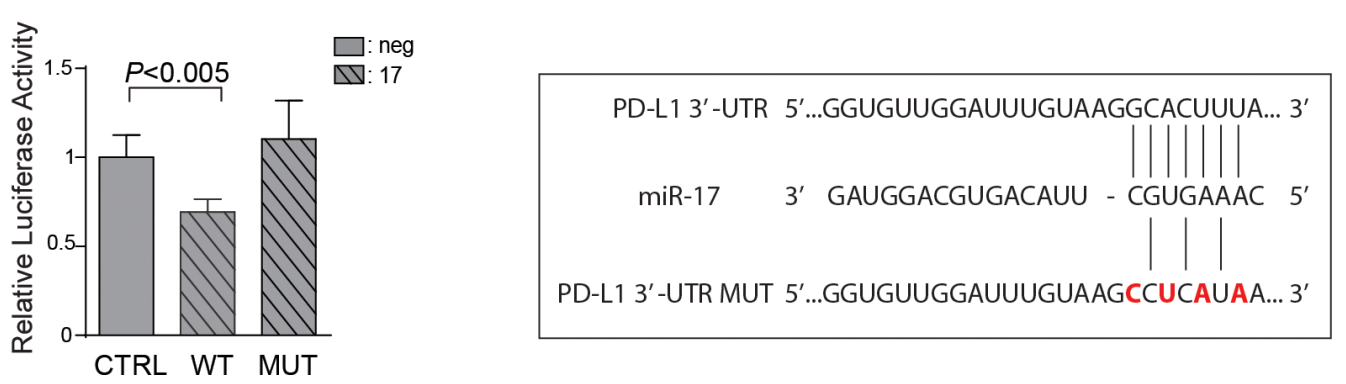

Figure 6: miR-17-5p regulates $C D 274 /$ PD-L1 expression. A. miR-17-5p basal expression in the sensitive (S) and BiR or MiR melanoma cells in A375, SKMEL5, M14 and A375/PD-L1 ${ }^{+}$. B. Pearson's correlation of miR-17-5p and PD-L1 level expression in the sensitive (S) and BiR or MiR melanoma cells in A375, SKMEL5, M14 and A375/PD-L1+. C. Relative expression of CD274/PD-L1 in miR-17-5p transfected cells in comparison to miR-negative transfected control in A375 and SKMEL5 cell line variants. D. Representative blot for PD-L1 protein of BiR or MiR of A375 and SKMEL5 transfected cells with pre-miR-17-5p (indicated as 17) and negative control (indicated as neg). E. Relative luciferase activity of $293 \mathrm{~T}$ cells transfected with miR-17-5p (indicated as 17) or negative control (indicated as neg) together with the luciferase vector containing PD-L1 3'-UTR wild type (WT) or mutagenized (MUT). F. Seeding sites of miR-17-5p in PD-L1 3'-UTR wild type and mutagenized (MUT). 
activity after 48 hours, suggesting a direct effect (Figure $6 \mathrm{E})$. Mutagenesis of miR-17-5p binding site in the 3'-UTR of PD-L1 mRNA (Figure 6F) failed to modulate luciferase activity, confirming specificity of binding between miR17-5p and the 3'-UTR of PD-L1 mRNA (Figure 6E).

In agreement with results obtained after silencing PD-L1, transfection of miR-17-5p in BiR and MiR melanoma cells was followed by a markedly decreased ability to repair wounds, indirectly validating the role of PD-L1 in this process (Figure 7A-7B). In these experimental conditions, miR17-5p levels rose sharply upon transfection (Supplementary Figure 3A).

\section{Expression of PD-L1 is acquired by BRAFi-} resistant melanoma lesions, is associated to aggressive behavior and unfavorable outcome and is inversely correlated with plasmatic miR17-5p levels

To obtain an independent validation of our in vitro data, we took advantage of a cohort of $80 \mathrm{BRAF}^{\mathrm{V} 600 \mathrm{E}}$ mutated MM patients, treated with BRAFi. Patient characteristics are reported in Supplementary Table 3. For each of these patients PD-L1 expression, as well as

A
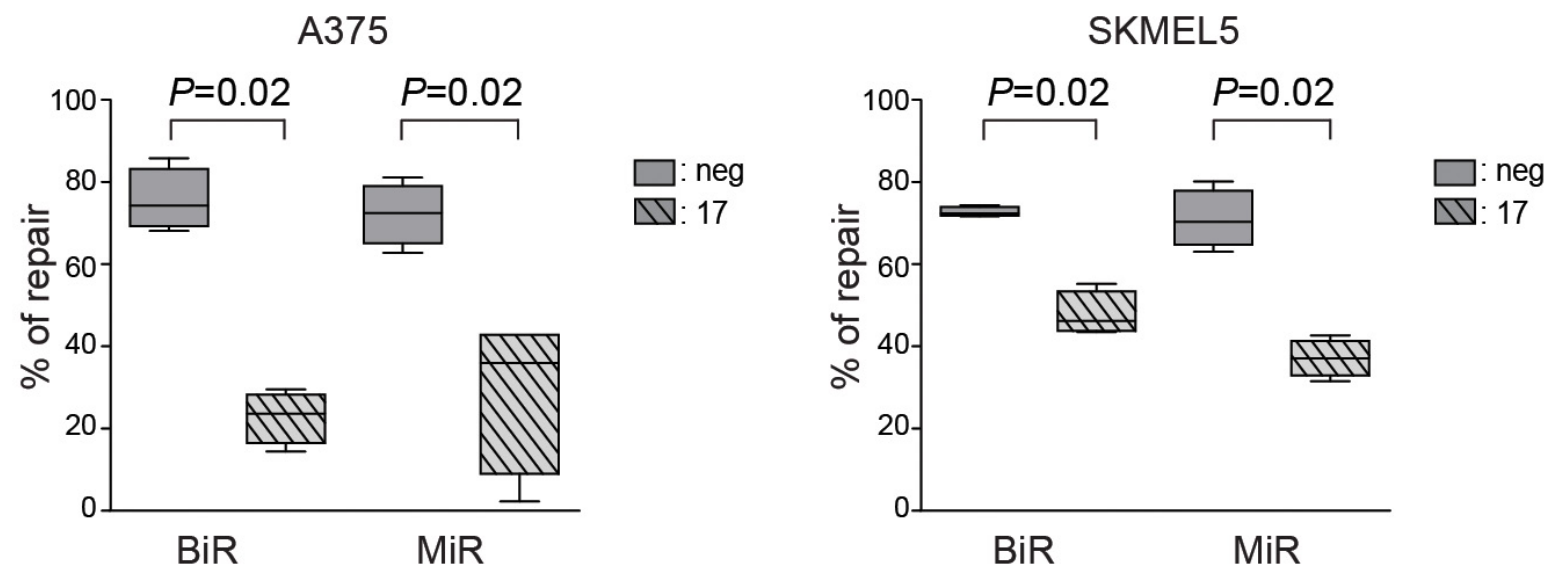

B
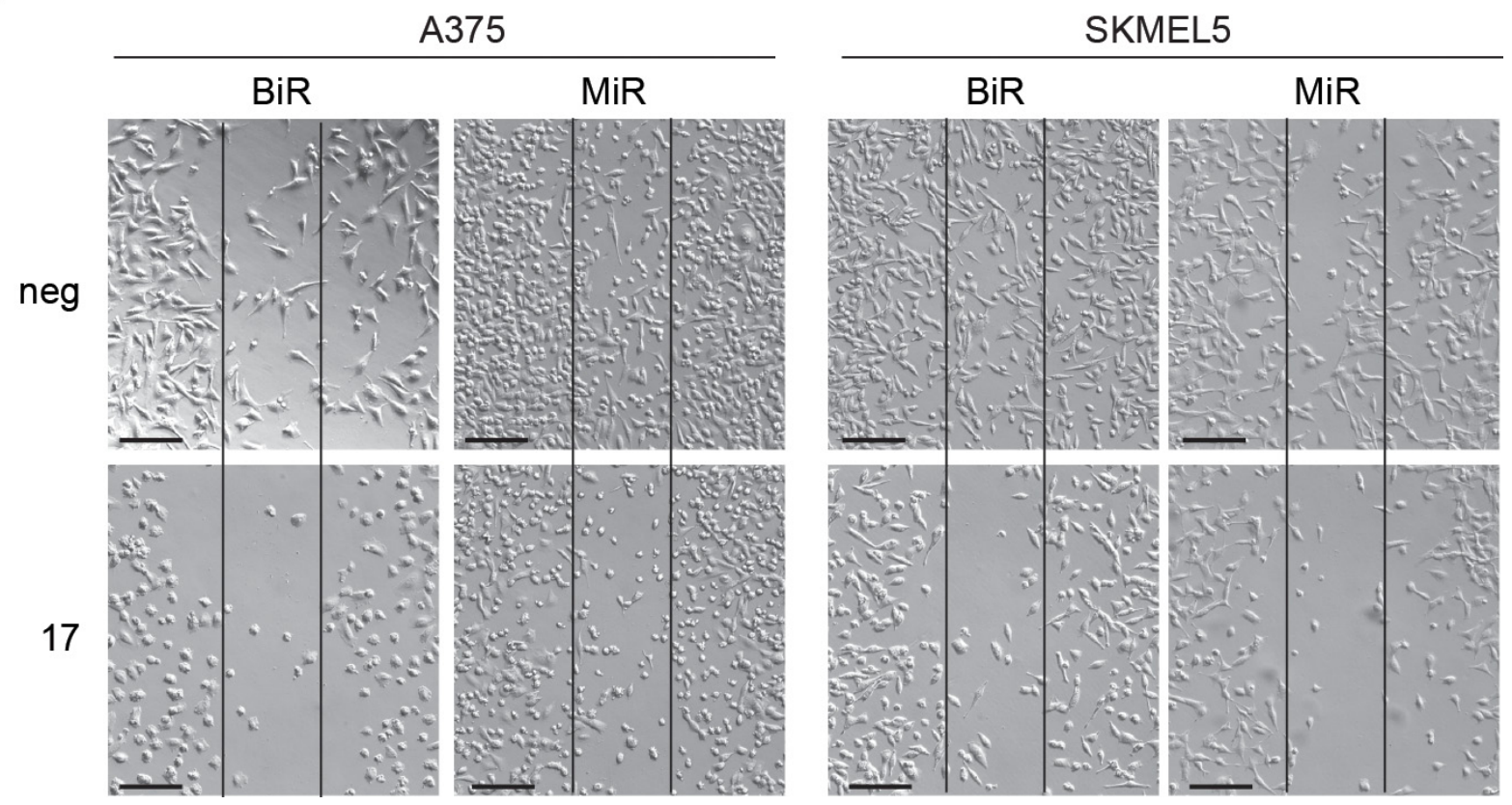

Figure 7: Modulation of PD-L1 can alter the functional properties of tumor cells. A. Quantification as percentage of repair of wound-healing assay comparing miR-negative transfected control and miR-17-5p transfected A375/BiR, A375/MiR, SKMEL5/BiR and SKMEL5/MiR. B. Representative pictures (x10 magnification, scale bar $200 \mu \mathrm{m}$ ) of A375/BiR, A375/MiR, SKMEL5/BiR and SKMEL5/ MiR. 
tumor infiltration by mononuclear cells (TIMC), were determined by immunohistochemistry. Data are available for 78 patients, because one PD-L1 ${ }^{+}$patient was not evaluable for TIMC and one patient without TIMC was not evaluable for PD-L1 expression.

Four different patient subsets were defined based on the combination of the two parameters. Of the $28 / 78$ patients with a PD-L1 ${ }^{+}$biopsy, 15 showed evidence of TIMC, suggesting that environmental factors, such as IFN $\gamma$ produced by activated $T$ cells, could be responsible for PD-L1 expression by the tumor cells. On the contrary, $13 / 28$ of PD-L1 ${ }^{+}$patients did not show TIMC, arguing in favor of a constitutive expression of PD-L1 in this subset. Of the 50/78 patients with a PD-L1- biopsy 14/50 had TIMC, while 36/50 did not have TIMC.

Our previous analysis of this cohort indicated that expression of PD-L1 by tumor cells and TIMC were two independent predictors of response to therapy with BRAFi $[27,31]$. We now updated results with a median followup of 27.4 months, by which time 45 (64\%) patients had progressed and 46 (52.5\%) had died. Overall, 55 (69\%) patients had either progressed or died, while 49 (61.3\%) patients reached a complete or partial response at one time during the course of their illness.

Multivariate analysis indicated that patients with a PD-L1 ${ }^{+}$biopsy and without TIMC were the least likely to respond to treatment and consequently those with the shortest overall survival. This finding is in line with the hypothesis that constitutive PD-L1 expression by the tumor identifies a subset of MM patients characterized by a highly aggressive disease. Patients with PD-L1 ${ }^{-}$biopsies and without TIMC (OR 15.69, 95\% CI 2.10-117.26, $P<$ 0.0073 ) or patients with PD-L1 ${ }^{-}$biopsies, but with TIMC (OR 17.26, 95\% CI 3.1-96.18, $P<0.0012$ ) showed a higher probability to have a complete or partial response compared to patients with $\mathrm{PD}-\mathrm{L1}^{+}$biopsies and without TIMC, while patients with $\mathrm{PD}-\mathrm{L1}^{+}$biopsies and with TIMC showed an intermediate behavior (Figure 8A and Supplementary Table 4). Furthermore, after adjusting for stage and performance status, lack of PD-L1 expression and presence of TIMC were associated to a significantly longer progression free survival (PFS, HR 0.37, 95\% CI $0.17-0.84, P<0.02$, Supplementary Table 5) and overall survival (OS, HR 0.35 95\% CI 0.15-0.84, $P<0.02$, Supplementary Table 6), compared to patients expressing PD-L1 and lacking TIMC. Kaplan Meier curves for PFS and OS are shown in Figure 8A and Supplementary Figure 4.

In order to evaluate whether PD-L1 could be dynamically modulated during BRAFi treatment, we obtained paired biopsies before beginning of therapy and after the onset of resistance to BRAFi (dabrafenib or vemurafenib) from 11 patients. Out of these 11 patients, in 7 cases (63\%, 95\% CI 39-94) we observed up-regulation of PD-L1 in melanoma cells by immunohistochemistry, as shown in three representative patients (Figure 8B-8C).
Specifically, in 4 cases with PD-L1' pre-treatment biopsies (failing to reach the $\geq 5 \%$ threshold) upon resistance we observed a shift from PD-L1- to $\mathrm{PD}-\mathrm{L1}^{+}$status, while in the remaining cases PD-L1 was highly up-regulated in resistant melanoma tissues when compared with baseline, with diffuse and uniform membranous pattern of expression, irrespective from the presence of infiltrating immune cells (Figure 8B-8C and Supplementary Table 7).

Interestingly, we were able to obtain paired biopsies in 4 patients who previously received a BRAF inhibitor monotherapy (dabrafenib) alone, and subsequently a combination of dabrafenib and trametinib. In these patients, who progressed during BRAFi and MEKi, we did not observe any modulation of PD-L1 expression (not shown), in agreement with previous data [32].

Lastly, we asked whether miR-17-5p levels in plasma from patients with metastatic melanoma could be used as an inverse marker of the expression of PD-L1 by the tumor. We collected sera from patients with metastatic melanoma before treatment with BRAFi. Patients were divided into two groups according to PD-L1 expression obtained by IHC: following this approach 16 patients were PD-L1 ${ }^{+}$and 6 PD-L1'. RT-PCR was used to quantify miR$17-5 \mathrm{p}$ levels in patient sera, showing that patients with a $\mathrm{PD}-\mathrm{L1}^{+}$tumor had lower levels of miR17-5p (mean \pm SD 34.1 \pm 3.4 ), compared to patients with a PD-L1- lesion (mean \pm SD 21.47 \pm 2.0 , Figure 8D).

\section{DISCUSSION}

The therapy of melanoma changed radically with the identification of recurrent mutations in genes coding for members of the MAPK pathway, which then led to the design of targeted inhibitors for both BRAF and MEK tyrosine kinases. Resistance to these drugs is a common event that is linked to the paradoxical activation of the MAP kinases axis, representing the limiting factor in this therapeutic approach. More recent studies are showing that the combination of tyrosine kinase inhibitors with immune checkpoint inhibitors, such as PD-L1, obtains more durable responses [33]. The rationale for these combinations is that MM frequently express PD-L1, which may be transcriptionally regulated by different signals. PD-L1 expression is dynamically modulated by IFN- $\gamma$, produced by infiltrating $\mathrm{CD}^{+}$activated $\mathrm{T}$ lymphocytes, representing an immune escape circuit. However, PD-L1 may be also expressed by melanoma cells in the absence of immune infiltrate, suggesting that expression relies on cell autonomous mechanisms.

The aim of this work was twofold. First, we wanted to expand our previous observations indicating that PDL1 is a marker for an aggressive form of melanoma by addressing the issue of whether the molecule may be directly involved in determining this phenotype. Secondly, we wanted to understand how PD-L1 expression is regulated in these cells. The data obtained in vitro were 


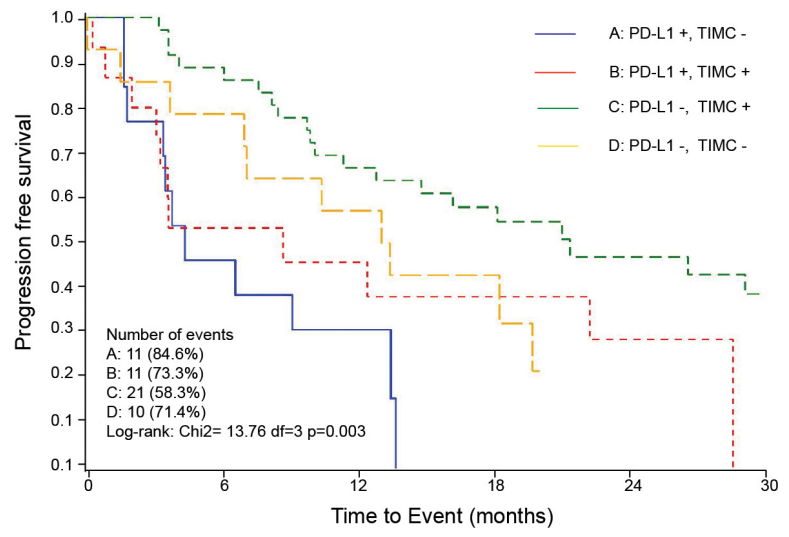

B
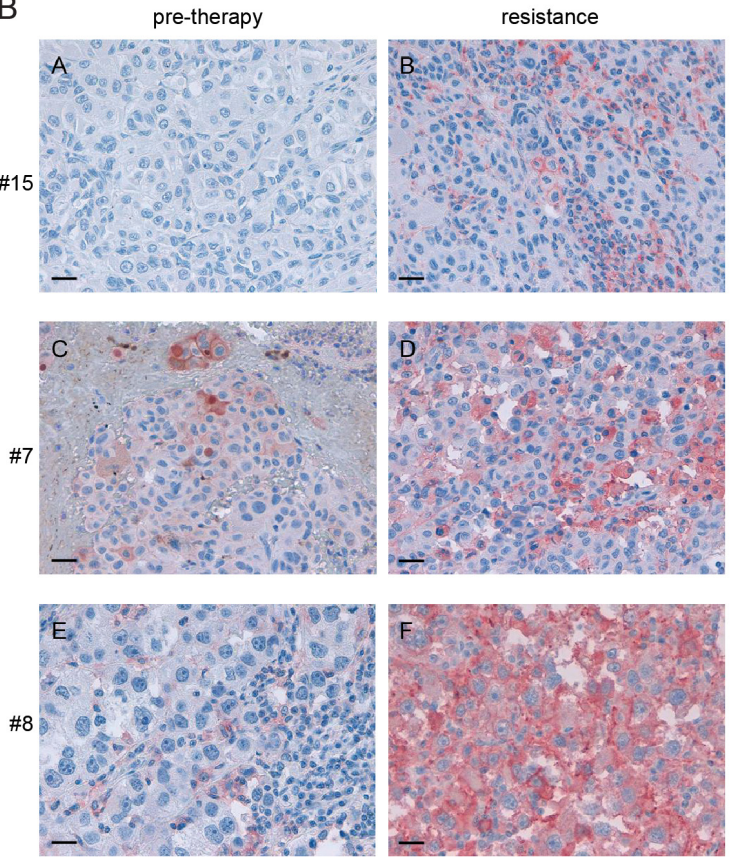

C

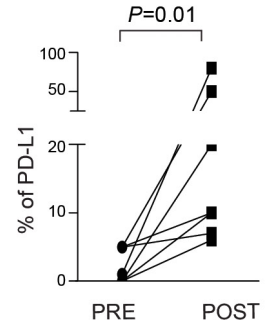

D

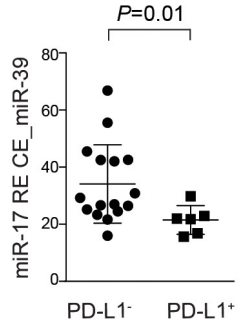

Figure 8: PD-L1 immunohistochemical expression in paired tissue biopsies taken before and after onset of resistance in patients treated with BRAF inhibitors (dabrafenib or vemurafenib). A. Survival curves estimated with the Kaplan-Meier method, on $\mathrm{y}$ axis is indicated the overall survival $\mathrm{OV}$, on the $\mathrm{x}$ axis is indicated the time to event in months. B. PD-L1 expression in three representative patients (pre-therapy and upon resistance acquisition conditions): patient \#15 (vemurafenib): PD-L1 is not expressed before resistance while it is observed in 6\% of tumor cells after resistance; patient \#7 (dabrafenib): PD-L1 positivity in 5\% of tumor cells before resistance and in 10\% of tumor cells upon resistance; patient \#8 (dabrafenib): tumor tissue before resistance is considered PD-L1', being observed only in $1 \%$ of tumor cells (cut-off $\geq 5 \%$ ), while PD-L1 is strongly and diffusely expressed in $80 \%$ of tumor cells upon resistance. Original magnification x 40, scale bar $50 \mu \mathrm{m}$. C. Percentage of PD-L1 immunostaining in tissue before resistance (PRE) and post resistance (POST) to BRAF inhibitors. D. Relative expression of miR-17-5p normalized on spike-in control miR-39 in serum blood patients before therapy. 
then validated using a cohort of melanoma patients with BRAF mutations, treated with BRAF inhibitors and where PD-L1 expression in the metastatic lesions had been studied.

After stabilizing BiR and MiR cells, we observed that these variants had a more aggressive behavior compared to the $\mathrm{S}$ counterparts. Resistance induced expression of a panel of common genes linked to cell movement. Functionally, BiR and MiR cells were characterized by enhanced wound repair, which could be due to the up-regulation of molecules that play an essential role in melanoma metastasis, including integrin family members. However, a direct role of PD-L1 was shown after observing decreased wound healing in BiR or MiR cells where PD-L1 had been stably silenced by lentiviral infection. Interestingly, these cells also showed a marked down-regulation in the expression of CD56, which was proposed as a marker of desmoplastic and spindle-cell melanomas. Even if rare, this is a kind of melanoma with highly invasive properties. Even if future studies are needed to determine whether these tumors are constitutively $\mathrm{PD}-\mathrm{L1}^{+}$, a recent report suggests that they are particularly sensitive to therapy with anti-PD1/PD-L1 antibodies (abstract ASCO 2015).

The second result of this work concerns the identification of a post-transcriptional regulation of PDL1 levels, obtained through the miR-17-5p circuit. Our data indicate direct binding of this microRNA to the 3'UTR of PD-L1. Furthermore, transfection of miR-17-5p decreases expression of PD-L1, as well as aggressiveness of the tumor. The role of miR-17-5p cluster in cancer is matter of intense investigation. Loss of heterozigosity at the miR-17-5p locus, has been observed in association with progression of different kinds of solid tumors [34]. Furthermore, the miR-17-92 cluster is deleted in a significant proportion of human cancers, including $20 \%$ of melanomas [35], suggesting an oncosuppressor role for this molecule. Our results would agree with this hypothesis, as BiR or MiR MM cell lines show a downregulation of this microRNA, as compared to sensitive cells. The molecular circuits responsible for miR-17$5 \mathrm{p}$ modulation remain to be determined. At the light of recent data indicating loss of PTEN in aggressive melanoma, characterized by impaired immune responses, it is tempting to speculate that loss of PTEN may also be followed by loss of miR-17-5p, with the consequent lack of post-transcriptional regulation in PD-L1 and the described over-activation of the Wnt- $\beta$ catenin Akt/PI3K pathways.

In the final part of the work, we exploited a large Italian cohort of MM patients that were treated with BRAFi or MEKi. From this analysis we draw three conclusions. The first is a confirmation that PD-L1 is an independent negative prognostic marker. The second is that, among $\mathrm{PD}^{-\mathrm{L1}^{+}}$patients those lacking TIMC are characterized by the worst outcome, suggesting that constitutive PD-L1 expression characterizes the most aggressive form of the disease. The third is that the acquisition of resistance to BRAFi induces expression of PD-L1 in the majority of cases. Lastly, comparison of miR-17-5p levels in plasma of patients with PD-L1 ${ }^{+}$MM biopsies show decreased plasmatic levels of miR-17-5p compared to patients with a PD-L1- lesion. If confirmed in larger cohorts, this finding suggests that miR-17-5p plasmatic levels may be used as inverse indicators of PD-L1 expression levels by the tumor. It will also be important to determine miR-17-5p levels in sequential samples and to correlate them with PDL1 expression levels in the corresponding biopsies.

\section{MATERIALS AND METHODS}

\section{Melanoma cell lines}

The A375, SKMEL5 and M14 BRAF ${ }^{\mathrm{V} 600 \mathrm{E}}$-mutated cell lines were originally obtained through the American Type Culture Collection (ATCC) and the BRAF mutational status confirmed by Sanger sequencing. Cells were cultured in RPMI-1640 + 10\% fetal calf serum and $100 \mathrm{IU} / \mathrm{ml}$ penicillin / streptomycin (all from Sigma, Milan, Italy, referred to as complete medium). BRAFiand MEKi-resistant melanoma cells (indicated as BiR and MiR) were generated by treating cells with increasing concentrations of BRAFi (dabrafenib, GlaxoSmithKline, Brentford, UK) or MEKi (pimasertib, Merk Serono, Darmstadt, Germany), reaching the final concentration of $1.6 \mu \mathrm{M}$ in $\approx 12$ weeks. Cells were thereafter maintained under these culture conditions $[23,36]$.

\section{MTT viability assay}

Cells were plated in complete medium and left overnight to attach to 96 -well plates. BRAFi or MEKi were then added at the indicated concentrations for 72 hours, before a 4-hour incubation with $10 \mu \mathrm{l}$ of (3-(4,5-di methylthiazolyl-2)-2,5 diphenyltetrazolium bromide, MTT, Thermo Scientific, Milan, Italy). The formazan crystals formed were dissolved in sodium dodecyl sulfatehydrochloride and absorbance was read at $570 \mathrm{~nm}$ using a microplate reader (Bio-Rad, Milan, Italy).

\section{Flow-cytometry}

Antibodies used for flow cytometry were: anti-PDL1-PE and -PE-Cy7, anti-CD24-APC, anti-CD56-PE, antiHLA-DR-PE-Cy7 (all from eBioscience, Milan, Italy), anti-integrin- $\alpha 3 \mathrm{mAb}$ (from Prof. G. Tarone, University of Turin, Italy). A secondary PE-conjugated goat anti-mouse Ig (Thermo Scientific, Milan, Italy) was used to highlight the binding of unlabeled antibodies.

Immunofluorescence data were acquired using a FACSCantoII cytofluorimeter and processed with DIVA v8.0 (BD Biosciences) and FlowJo Version 9.01 softwares 
(TreeStar, Ashland, OR, USA), analyzing at least 10,000 events per sample.

\section{Western blot analysis}

Cells were lysed, resolved by SDS-PAGE, and transferred to nitrocellulose filter membranes (Biorad, Milan) [27]. After blocking, membranes were incubated with: anti-PD-L1 (R\&D Systems, Milan, Italy), -pSTAT3, -STAT3, -pERK1/2 and -ERK1/2 (all from Cell Signaling Technology, Danvers, MA). After incubation with horseradish peroxidase-conjugated secondary antibody (PerkinElmer, Milan, Italy), reaction was visualized with ECL using ImageQuant LAS4000 (GE Healthcare, Milan, Italy).

\section{Confocal microscopy}

Cells were cultured overnight on glass cover slips in 24-well plates before incubation with unlabeled antiPD-L1 antibody (eBioscience), followed by AlexaFluor488-conjugated goat anti-mouse IgG (Thermo Scientific). Phalloidin AlexaFluor-568-conjugated and DAPI (both from Thermo Scientific) were added after fixation (4\% paraformaldehyde) and permeabilization ( $0.1 \%$ saponin). Slides were then analyzed using a TCS SP5 laser scanning confocal microscope equipped with 4 lasers and images were acquired with LAS AF Version Lite 2.4 software (Leica Microsystems, Wetzlar, Germany), as described [37]. Files were processed with Photoshop (Adobe Systems, San Jose, CA).

\section{Wound healing assay}

Melanoma cells were seeded in 6-well plates $\left(5 \times 10^{5} /\right.$ well $)$ and incubated in complete medium overnight. Cells were then treated with Mitomycin C (10 $\mu \mathrm{g}, 20$ minutes, Sigma). Wounds were made with a $200 \mu \mathrm{l}$ tip and the wells were washed several times to remove all non-adherent cells. Wound repair was documented at 24 hours using a DMI 3000 B optical microscope (Leica Microsystems), equipped with a DCF 310 FX digital camera and LAS Version 3.8 software. Images were analyzed with MRI Wound Healing Tool of ImageJ software (NIH, USA). The percentage of repair was calculated as: [( $\left.\left.\operatorname{area}_{24 \text { hours }} / \operatorname{area}_{0 \text { hours }}\right) \times 100 \%\right]$.

\section{Chemotaxis and invasion assays}

Migration and invasion were measured using $8 \mu \mathrm{M}$ pore Boyden chambers (Corning, Corning, NY). Briefly, $10^{5}$ cells were plated in the upper chamber in serum free RPMI-1640 medium, while complete medium was added as a chemoattractant in the lower part. After 4 hours, cells in the upper part of the chamber were removed, while cells that had migrated to the lower surface of the filter stained with crystal violet (Sigma) and analyzed by bright-field microscopy. Migration index was calculated as: number of cells migrated in the presence of the chemoattractant / number of cells migrated without chemoattractant.

Invasion assays were performed after covering the upper part of the well with Matrigel $(0.5 \mathrm{mg} / \mathrm{ml}$, Corning). After 24 hours, cells that had not penetrated were wiped away, while cells that had invaded the lower surface of the filter were stained with crystal violet and examined by bright-field microscopy [38]. Invasion index was calculated as: number of cells penetrated in the presence of the chemoattractant / number of cells penetrated without chemoattractant.

\section{Xenograft models}

A375/S and A375/BiR cells $\left(10^{7}\right)$ were injected subcutaneously in the presence of Matrigel into the right and left flanks, respectively, of 6- to 8-week-old male NOD/SCID mice. When tumors became palpable $(\approx$ after two weeks), mice were treated with BRAFi (30 mg/kg) daily by gavage for 1 week (Tafinlar, Novartis, Basel, Switzerland). After treatment, animals were sacrificed and tumors measured. Lesions were then partly fixed and processed for histopathological studies and partly dissociated for cytofluorimetric analyses and cultures. Recultured cells were tested to confirm resistance to BRAFi by the MTT assay.

\section{Quantitative real-time PCR (qRT-PCR)}

Total RNA (containing mRNAs and microRNAs) was extracted using miRNeasy kit (Qiagen, Milan, Italy) and converted to cDNA using the High Capacity cDNA Reverse Transcription kit (Thermo Scientific). qRT-PCR was performed using the 7900 HT Fast Real Time PCR system (SDS2.3 software) using commercially available primers (all from Thermo Scientific) and standardized over actin levels. Reactions were done in triplicate from the same cDNA reaction (technical replicates). Detection of microRNAs was performed by qRT-PCR for the specific hsa-miR and U6 snRNA (ID 001973, Thermo Scientific), as control for the cell lines. For normalization in blood sera from patients miR-39 from C. elegans [39] was added during the extraction of microRNAs. The comparative CT method was used to calculate the relative expression of the gene under analysis.

TargetScan 6.0 algorithm was used to identify predicted microRNA targets that bind 3'-UTR of PD-L1 mRNA.

\section{RNA sequencing (RNA-seq)}

RNA-seq was performed as previously described [40], with few modifications. Briefly, the DNF-471 Standard Sensitivity RNA Analysis Kit, run on Fragment Analyzer (both from Advanced Analytical, Ankeny IA) was used to check RNA quality. Libraries were prepared 
from total RNA using TruSeq RNA Sample Preparation v2 according to the manufacturer's protocol (Illumina, San Diego, CA). Samples were sequenced on Illumina HiScanSQ platform. Sequencing reads were trimmed out of the low-quality bases with Fastx Toolkit (HannonLab, CHSL). Filtered sequences were mapped on hg19 genome assembly by using TopHat v2.0.6 and mRNA quantification was performed using Cuffdiff v2.0.2. For downstream analysis, genes with RPKM $<1$ in all the samples were filtered out. Custom scripts on R software were used for clustering and heatmap analysis (https://www.r-project. org/). Gene Ontology was analyzed by using Database for Annotation, Visualization and Integrated Discovery (DAVID) program (https://david.ncifcrf.gov/).

\section{Transient transfections}

Melanoma cell lines were transfected with pre-miR17-5p microRNA precursor molecules and microRNA negative control (Thermo Scientific) using HiPerFect Transfection Reagent (Qiagen). Expression of miRs or protein-coding genes overexpression/knockdown was monitored from 24 to 96 hours later by qRT-PCR.

\section{Luciferase assays}

Luciferase reporter vectors containing the partial PDL1 3'-UTR were generated following PCR amplification (forward primer: CAGGCAAGAATTGTGGCTGA, reverse primer: CCAAGTAACTTTCTCCACTGGGAT) of the 3'-UTR from human genomic DNA of PD-L1 ${ }^{+}$cells and cloning into the Firefly Luciferase reporter pMIR REPORT $^{\mathrm{TM}}$ luciferase vector (Thermo Scientific). When indicated the 3'-UTR was mutagenized at the miR-17$5 \mathrm{p}$ recognition site using the QuickChange Site-Directed Mutagenesis kit (Stratagene, Cedar Creek, TX) according to the manufacturer's instructions (forward primer: GA TGAAACATGAGACAAAAGGGATTATGAGGCTT ACAAATCCAACACCACAAGGA, reverse primer: TC CTTGTGGTGTTGGATTTGTAAGCCTCATAATCCC TTTTGTCGCATGTTTCATC).

Cells $\left(5 \times 10^{4}\right)$ were then co-transfected with 50 ng of the pMIR REPORT ${ }^{\text {TM }}$ (Thermo Scientific) Firefly Luciferase constructs containing the 3'-UTRs of the specific microRNA potential target, $20 \mathrm{ng}$ of pRL-TK Renilla Luciferase normalization control (Promega, Madison, WI) and $75 \mathrm{nM}$ of the indicated pre-miR using Effectene (Qiagen). Lysates were collected 48 hours after transfection and Firefly and Renilla Luciferase activities were measured with a Dual-Luciferase Reporter System (Promega).

\section{Preparation of lentiviruses}

Lentiviral particles containing the genetic material for shPD-L1 (Origene, Rockville, MD) were generated according to the manufacture's protocol.
Resistant cell lines were infected with shPD-L1 lentiviral particles and expression of the molecule monitored 48 hours later by flow cytometry. To obtain stably PD-L1-silenced clones from A375 or SKMEL5 / $\mathrm{BiR}$ or /MiR lines, infected cells were repeatedly sorted with a BD FACSAriaIII (BD Biosciences) by gating on $\mathrm{GFP}^{+} / \mathrm{PD}-\mathrm{L} 1$ -

\section{PD-L1 immunohistochemistry in human melanoma tissues}

Cohort characteristics and detailed immunohistochemical protocols were previously described [31].

\section{Statistical analyses}

Continuous variables were compared by MannWhitney test. The Wilcoxon matched-pairs signed rank test was used for paired variables.

For the clinical study, all melanoma patients satisfying eligibility criteria and treated with BRAFi were considered for analysis. Overall response rate (ORR) was defined as the proportion of patients with complete response or partial response, according to RECIST, v1.1 [41]. Progression free survival (PFS) was defined as the time from the beginning of BRAFi to first appearance of progressive disease or death for any cause; patients known to be alive and without progressive disease at the time of analysis were censored at their last available follow-up assessment. Overall survival (OS) was defined as the time from the beginning of BRAFi to the date of death from any cause or the date of the last follow-up. Survival curves were estimated with the Kaplan-Meier method. PFS and OS were analyzed by means of Cox regression model and results were expressed as hazard ratios (HR) with their 95\% confidence intervals (95\% CI) ORR was analyzed by means of logistic regression models and results were expressed as odds ratios (OR) with their $95 \% \mathrm{CI}$.

Statistical analyses were performed using SAS software, version 9.2 (SAS Institute, Cary, NC) and GraphPad version 6 (GraphPad Software Inc, La Jolla, CA).

\section{ACKNOWLEDGMENTS}

Thanks are given to Katiuscia Gizzi for excellent technical support. The contribution of Dr. Davide Brusa in the generation of BiR and MiR cell lines is acknowledged.

\section{CONFLICTS OF INTEREST}

The authors declare no conflicts of interest.

\section{GRANT SUPPORT}

Human Genetics Foundation Institutional funds, University of Turin research grants (ex-60\% to SD), 
Associazione Italiana per la Ricerca sul Cancro AIRC Investigator grants (IG-17314 to SD and IG-15217 to SO) and Fondazione Ente Cassa di Risparmio di Firenze grant \#2014.0240 to DM.

VA is supported by FIRC/AIRC triennial (\#15047) and "Carlo Chianello" Foundation fellowships.

\section{REFERENCES}

1. Chapman PB, Hauschild A, Robert C, Haanen JB, Ascierto P, Larkin J, Dummer R, Garbe C, Testori A, Maio M, Hogg D, Lorigan P, Lebbe C, Jouary T, Schadendorf D, Ribas A, et al. Improved survival with vemurafenib in melanoma with BRAF V600E mutation. N Engl J Med. 2011; $364: 2507-2516$

2. Hauschild A, Grob JJ, Demidov LV, Jouary T, Gutzmer R, Millward M, Rutkowski P, Blank CU, Miller WH, Jr., Kaempgen E, Martin-Algarra S, Karaszewska B, Mauch C, Chiarion-Sileni V, Martin AM, Swann S, et al. Dabrafenib in BRAF-mutated metastatic melanoma: a multicentre, open-label, phase 3 randomised controlled trial. Lancet. 2012; 380:358-365.

3. Flaherty KT, Infante JR, Daud A, Gonzalez R, Kefford RF, Sosman J, Hamid O, Schuchter L, Cebon J, Ibrahim N, Kudchadkar R, Burris HA, 3rd, Falchook G, Algazi A, Lewis $\mathrm{K}$, Long GV, et al. Combined BRAF and MEK inhibition in melanoma with BRAF V600 mutations. N Engl J Med. 2012; 367:1694-1703.

4. Hertzman Johansson C, Egyhazi Brage S. BRAF inhibitors in cancer therapy. Pharmacol Ther. 2014; 142:176-182.

5. Girotti MR, Lopes F, Preece N, Niculescu-Duvaz D, Zambon A, Davies L, Whittaker S, Saturno G, Viros A, Pedersen M, Suijkerbuijk BMJM, Menard D, McLeary R, Johnson L, Fish L, Ejiama S, et al. Paradox-Breaking RAF Inhibitors that Also Target SRC Are Effective in DrugResistant BRAF Mutant Melanoma. Cancer Cell. 2015; 27:85-96.

6. Lugowska I, Kosela-Paterczyk H, Kozak K, Rutkowski P. Trametinib: a MEK inhibitor for management of metastatic melanoma. Onco Targets Ther. 2015; 8:2251-2259.

7. Long GV, Stroyakovskiy D, Gogas H, Levchenko E, de Braud F, Larkin J, Garbe C, Jouary T, Hauschild A, Grob JJ, Chiarion Sileni V, Lebbe C, Mandala M, Millward M, Arance A, Bondarenko I, et al. Combined BRAF and MEK inhibition versus BRAF inhibition alone in melanoma. N Engl J Med. 2014; 371:1877-1888.

8. Larkin J, Ascierto PA, Dreno B, Atkinson V, Liszkay G, Maio M, Mandala M, Demidov L, Stroyakovskiy D, Thomas L, de la Cruz-Merino L, Dutriaux C, Garbe C, Sovak MA, Chang I, Choong N, et al. Combined vemurafenib and cobimetinib in BRAF-mutated melanoma. N Engl J Med. 2014; 371:1867-1876.

9. Robert C, Karaszewska B, Schachter J, Rutkowski P, Mackiewicz A, Stroiakovski D, Lichinitser M, Dummer R,
Grange F, Mortier L, Chiarion-Sileni V, Drucis K, Krajsova I, Hauschild A, Lorigan P, Wolter P, et al. Improved overall survival in melanoma with combined dabrafenib and trametinib. N Engl J Med. 2015; 372:30-39.

10. Ramsay AG. Immune checkpoint blockade immunotherapy to activate anti-tumour T-cell immunity. Br J Haematol. 2013; 162:313-325.

11. Day CL, Kaufmann DE, Kiepiela P, Brown JA, Moodley ES, Reddy S, Mackey EW, Miller JD, Leslie AJ, DePierres C, Mncube Z, Duraiswamy J, Zhu B, Eichbaum Q, Altfeld $\mathrm{M}$, Wherry EJ, et al. PD-1 expression on HIV-specific T cells is associated with T-cell exhaustion and disease progression. Nature. 2006; 443:350-354.

12. Blank C, Gajewski TF, Mackensen A. Interaction of PD-L1 on tumor cells with PD-1 on tumor-specific T cells as a mechanism of immune evasion: implications for tumor immunotherapy. Cancer Immunol Immunother. 2005; 54:307-314.

13. Zheng $P$, Zhou Z. Human Cancer Immunotherapy with PD-1/PD-L1 Blockade. Biomark Cancer. 2015; 7:15-18.

14. Robert C, Long GV, Brady B, Dutriaux C, Maio M, Mortier L, Hassel JC, Rutkowski P, McNeil C, Kalinka-Warzocha E, Savage KJ, Hernberg MM, Lebbe C, Charles J, Mihalcioiu C, Chiarion-Sileni V, et al. Nivolumab in previously untreated melanoma without BRAF mutation. N Engl J Med. 2015; 372:320-330.

15. Ribas A, Puzanov I, Dummer R, Schadendorf D, Hamid O, Robert C, Hodi FS, Schachter J, Pavlick AC, Lewis KD, Cranmer LD, Blank CU, O'Day SJ, Ascierto PA, Salama AK, Margolin KA, et al. Pembrolizumab versus investigator-choice chemotherapy for ipilimumab-refractory melanoma (KEYNOTE-002): a randomised, controlled, phase 2 trial. Lancet Oncol. 2015; 16:908-918.

16. Weber JS, D'Angelo SP, Minor D, Hodi FS, Gutzmer R, Neyns B, Hoeller C, Khushalani NI, Miller WH, Jr., Lao CD, Linette GP, Thomas L, Lorigan P, Grossmann KF, Hassel JC, Maio M, et al. Nivolumab versus chemotherapy in patients with advanced melanoma who progressed after anti-CTLA-4 treatment (CheckMate 037): a randomised, controlled, open-label, phase 3 trial. Lancet Oncol. 2015; 16:375-384.

17. Larkin J, Chiarion-Sileni V, Gonzalez R, Grob JJ, Cowey CL, Lao CD, Schadendorf D, Dummer R, Smylie M, Rutkowski P, Ferrucci PF, Hill A, Wagstaff J, Carlino MS, Haanen JB, Maio M, et al. Combined Nivolumab and Ipilimumab or Monotherapy in Untreated Melanoma. N Engl J Med. 2015; 373:23-34.

18. Postow MA, Chesney J, Pavlick AC, Robert C, Grossmann K, McDermott D, Linette GP, Meyer N, Giguere JK, Agarwala SS, Shaheen M, Ernstoff MS, Minor D, Salama AK, Taylor M, Ott PA, et al. Nivolumab and ipilimumab versus ipilimumab in untreated melanoma. N Engl J Med. 2015; 372:2006-2017. 
19. Blank C, Brown I, Peterson AC, Spiotto M, Iwai Y, Honjo T, Gajewski TF. PD-L1/B7H-1 inhibits the effector phase of tumor rejection by $\mathrm{T}$ cell receptor (TCR) transgenic CD8+ T cells. Cancer Res. 2004; 64:1140-1145.

20. Lee SJ, Jang BC, Lee SW, Yang YI, Suh SI, Park YM, Oh S, Shin JG, Yao S, Chen L, Choi IH. Interferon regulatory factor-1 is prerequisite to the constitutive expression and IFN-gamma-induced upregulation of B7-H1 (CD274). FEBS Lett. 2006; 580:755-762.

21. Taube JM, Anders RA, Young GD, Xu H, Sharma R, McMiller TL, Chen S, Klein AP, Pardoll DM, Topalian SL, Chen L. Colocalization of inflammatory response with B7-h1 expression in human melanocytic lesions supports an adaptive resistance mechanism of immune escape. Sci Transl Med. 2012; 4:127ra137.

22. Spranger S, Spaapen RM, Zha Y, Williams J, Meng Y, Ha TT, Gajewski TF. Up-regulation of PD-L1, IDO, and $\mathrm{T}$ (regs) in the melanoma tumor microenvironment is driven by CD8(+) T cells. Sci Transl Med. 2013; 5:200ra116.

23. Jiang X, Zhou J, Giobbie-Hurder A, Wargo J, Hodi FS. The activation of MAPK in melanoma cells resistant to BRAF inhibition promotes PD-L1 expression that is reversible by MEK and PI3K inhibition. Clin Cancer Res. 2013; 19:598-609.

24. Atefi M, Avramis E, Lassen A, Wong DJ, Robert L, Foulad D, Cerniglia M, Titz B, Chodon T, Graeber TG, CominAnduix B, Ribas A. Effects of MAPK and PI3K pathways on PD-L1 expression in melanoma. Clin Cancer Res. 2014; 20:3446-3457.

25. Song M, Chen D, Lu B, Wang C, Zhang J, Huang L, Wang X, Timmons CL, Hu J, Liu B, Wu X, Wang L, Wang J, Liu H. PTEN loss increases PD-L1 protein expression and affects the correlation between PD-L1 expression and clinical parameters in colorectal cancer. PLoS One. 2013; 8:e65821.

26. Mittendorf EA, Philips AV, Meric-Bernstam F, Qiao N, Wu Y, Harrington S, Su X, Wang Y, Gonzalez-Angulo AM, Akcakanat A, Chawla A, Curran M, Hwu P, Sharma P, Litton JK, Molldrem JJ, et al. PD-L1 expression in triple-negative breast cancer. Cancer Immunol Res. 2014; 2:361-370.

27. Massi D, Brusa D, Merelli B, Ciano M, Audrito V, Serra S, Buonincontri R, Baroni G, Nassini R, Minocci D, Cattaneo L, Tamborini E, Carobbio A, Rulli E, Deaglio S, Mandala M. PD-L1 marks a subset of melanomas with a shorter overall survival and distinct genetic and morphological characteristics. Ann Oncol. 2014; 25:2433-2442.

28. Melchiori A, Mortarini R, Carlone S, Marchisio PC, Anichini A, Noonan DM, Albini A. The alpha 3 beta 1 integrin is involved in melanoma cell migration and invasion. Exp Cell Res. 1995; 219:233-242.

29. Tang MR, Wang YX, Guo S, Han SY, Li HH, Jin SF. CD24 expression predicts poor prognosis for patients with cutaneous malignant melanoma. Int J Clin Exp Med. 2014; 7:4337-4341.

30. Huttenbach Y, Prieto VG, Reed JA. Desmoplastic and spindle cell melanomas express protein markers of the neural crest but not of later committed stages of Schwann cell differentiation. J Cutan Pathol. 2002; 29:562-568.

31. Massi D, Brusa D, Merelli B, Falcone C, Xue G, Carobbio A, Nassini R, Baroni G, Tamborini E, Cattaneo L, Audrito V, Deaglio S, Mandala M. The status of PD-L1 and tumorinfiltrating immune cells predict resistance and poor prognosis in BRAFi-treated melanoma patients harboring mutant BRAFV600. Ann Oncol. 2015; 26:1980-1987.

32. Wargo JA, Cooper ZA, Flaherty KT. Universes collide: combining immunotherapy with targeted therapy for cancer. Cancer Discov. 2014; 4:1377-1386.

33. Ribas A, Flaherty KT. Gauging the Long-Term Benefits of Ipilimumab in Melanoma. J Clin Oncol. 2015; 33:1865-1866.

34. Coller HA, Forman JJ, Legesse-Miller A. "Myc'ed messages": myc induces transcription of E2F1 while inhibiting its translation via a microRNA polycistron. PLoS Genet. 2007; 3:e146.

35. Zhang L, Huang J, Yang N, Greshock J, Megraw MS, Giannakakis A, Liang S, Naylor TL, Barchetti A, Ward MR, Yao G, Medina A, O'Brien-Jenkins A, Katsaros D, Hatzigeorgiou A, Gimotty PA, et al. microRNAs exhibit high frequency genomic alterations in human cancer. Proc Natl Acad Sci U S A. 2006; 103:9136-9141.

36. Vergani E, Vallacchi V, Frigerio S, Deho P, Mondellini P, Perego P, Cassinelli G, Lanzi C, Testi MA, Rivoltini L, Bongarzone I, Rodolfo M. Identification of MET and SRC activation in melanoma cell lines showing primary resistance to PLX4032. Neoplasia. 2011; 13:1132-1142.

37. Audrito V, Serra S, Brusa D, Mazzola F, Arruga F, Vaisitti T, Coscia M, Maffei R, Rossi D, Wang T, Inghirami G, Rizzi M, Gaidano G, Garcia JG, Wolberger C, Raffaelli N, et al. Extracellular nicotinamide phosphoribosyltransferase (NAMPT) promotes M2 macrophage polarization in chronic lymphocytic leukemia. Blood. 2015; 125:111-123.

38. Righi L, Deaglio S, Pecchioni C, Gregorini A, Horenstein AL, Bussolati G, Sapino A, Malavasi F. Role of CD31/ platelet endothelial cell adhesion molecule-1 expression in in vitro and in vivo growth and differentiation of human breast cancer cells. Am J Pathol. 2003; 162:1163-1174.

39. Mitchell PS, Parkin RK, Kroh EM, Fritz BR, Wyman SK, Pogosova-Agadjanyan EL, Peterson A, Noteboom J, O'Briant KC, Allen A, Lin DW, Urban N, Drescher CW, Knudsen BS, Stirewalt DL, Gentleman R, et al. Circulating microRNAs as stable blood-based markers for cancer detection. Proc Natl Acad Sci U S A. 2008; 105:10513-10518.

40. Piva R, Deaglio S, Fama R, Buonincontri R, Scarfo I, Bruscaggin A, Mereu E, Serra S, Spina V, Brusa D, Garaffo 
G, Monti S, Dal Bo M, Marasca R, Arcaini L, Neri A, et al. The Kruppel-like factor 2 transcription factor gene is recurrently mutated in splenic marginal zone lymphoma. Leukemia. 2015; 29:503-507.

41. Therasse P, Arbuck SG, Eisenhauer EA, Wanders J, Kaplan RS, Rubinstein L, Verweij J, Van Glabbeke M, van Oosterom AT, Christian MC, Gwyther SG. New guidelines to evaluate the response to treatment in solid tumors. European Organization for Research and Treatment of Cancer, National Cancer Institute of the United States, National Cancer Institute of Canada. J Natl Cancer Inst. 2000; 92:205-216. 